Collection SFN 12 (2011) 123-159

(C) Owned by the authors, published by EDP Sciences, 2011

DOI: $10.1051 / \mathrm{sfn} / 201112007$

\title{
Introduction to the theory of lattice dynamics
}

\author{
M.T. Dove*
} Department of Earth Sciences, University of Cambridge, Downing Street,
Cambridge CB1 8BL, UK

\begin{abstract}
We review the theory of lattice dynamics, starting from a simple model with two atoms in the unit cell and generalising to the standard formalism used by the scientific community today. The key component of the theory is the force between atoms, and we discuss how this can be computed from empirical or quantum mechanical models. The basic model is developed to link the amplitudes of vibrations to thermodynamics. The major method for measuring lattice dynamics is inelastic scattering of beams of neutrons and x-rays. We develop a simplified theory of inelastic scattering of radiation beams, and show how this can be used in instrumentation. Several examples are used to illustrate the theory.
\end{abstract}

\section{INTRODUCTION}

\subsection{The study of lattice dynamics}

The subject of lattice dynamics is the study of the vibrations of the atoms in a crystal. Whilst we intuitively understand that atoms must be vibrating within crystals - it is the natural interpretation of temperature - traditional crystallography often leads to the image of atoms being held in static positions through stiff chemical bonds. Yet crystallographic measurements tell us that atoms can be vibrating with an amplitude that can be of order of $10 \%$ of an interatomic distance. Thus we need to understand lattice dynamics in order to have a complete picture of crystalline materials, and indeed of amorphous materials too. ${ }^{1}$

Understanding lattice dynamics is important for a number of key applications. The propagation of sound waves in crystals are a practical example of the role of lattice dynamics, as also is the interaction of materials with light. For example, the absorption of certain frequencies in the infra-red spectral region is directly due to the existence of specific lattice dynamics motions. Lattice dynamics also gives us properties such as thermodynamics, superconductivity, phase transitions, thermal conductivity, and thermal expansion.

In the study of lattice dynamics, atomic motions are frequently found to be adequately described as harmonic travelling waves. Each wave can be fully characterised in terms of its wavelength, $\lambda$, angular frequency, $\omega$, amplitude and direction of travel. Actually the wavelength is slightly hard to deal with, because we have values ranging in scale from infinity down to the distances of interatomic spacings. It is rather easier to use wave vector $\mathbf{k}$ instead, defined as the vector parallel to the direction of propagation of the wave, and normalised such that $|\mathbf{k}|=2 \pi / \lambda$. Moreover, we will see that there are conservation laws that are much easier to handle in terms of $\mathbf{k}$ rather than wavelength. The price we pay for this is that we need to think in terms of reciprocal space rather than real space. ${ }^{2}$ We will find that the $\omega$ is a

\footnotetext{
*e-mail: mtd10@cam.ac.uk
}

This is an Open Access article distributed under the terms of the Creative Commons Attribution-Noncommercial License 3.0, which permits unrestricted use, distribution, and reproduction in any noncommercial medium, provided the original work is properly cited.

1 This brief review can, at best, only scratch over the surface in an illustrative manner. The author has written two books that contain more detail $[1,2]$. Many books with a title containing phrases such as "solid state physics" will have a couple of chapters or so devoted to lattice dynamics, but beware of books whose treatment is more-or-less restricted to metals, because you will get a very incomplete story.

${ }^{2}$ For readers who need a basic course on the reciprocal lattice, I refer you to Chapter 4 of [2]. 
function of both $\mathbf{k}$ and the forces between atoms, and the amplitude of any wave is a function of $\omega$ and temperature.

The purpose of this paper is to discuss how our understanding of lattice dynamics is formulated in terms of travelling waves, together with the role of the interatomic forces. We will show how the formalism lends itself to practical computations based on models for the forces between atoms, and will show some practical examples of recent calculations. Moreover, neutron scattering provides an excellent tool for measuring lattice dynamics, and we will discuss this with key examples.

\subsection{Historical background}

Understanding something of the historical context behind any topic in science is often useful because it gives a perspective on the origin of the subject and where it may be heading. Thus the story of lattice dynamics begins in 1905, with Einstein's confirmation, via his theory of Brownian motion, that atoms really do exist [3, 4]. Within two years, Einstein had shown, using Planck's theory of radiation, that the temperature-dependence of the heat capacity of a solid could be explained through the quantisation of atomic vibrations [5]. However, these vibrations were merely postulated and not described, and hence were treated as simple vibrations with a single average frequency. In 1912 Born and von Kàrmàn created the model for lattice dynamics that introduced all the key components that are the foundation of the modern theory of lattice dynamics [6,7]. At the same time Debye introduced a model for the specific heat of a material that extended Einstein's approach by formulating the lattice dynamics in terms of sound waves [11]. Soon after it was shown - by Debye [8] and Waller [9] amongst others - that atomic vibrations had a significant effect on the intensity of Bragg reflections in x-ray crystallography, which had quickly become the established technique for deducing the atomic structure of materials. Each of these authors now have their names attached to components of the theory of lattice dynamics.

After this starting point very little happened in the field until the 1960 's. ${ }^{3}$ This was mostly due to the difficulty in applying the theory to real systems, and in the lack of experimental data. The early 1960 's saw the development and refinement of the use of neutron scattering to measure vibrational frequencies, and in particular the development of the triple-axis spectrometer as the primary piece of instrumentation (described later in Section 5.3). Simultaneously was the development of the computer and programming languages, which on one hand made routine lattice dynamics calculations feasible (including calculations to go hand-in-hand with experiments; methods are described later in Section 2.5), and on the other hand enabled experiments to be automated rather than having each one to be run as a monumental tour de force. The first examples, combining measurements with the triple-axis spectrometer and calculations, included studies of the lattice dynamics of simple metals and simple ionic materials. Thus inelastic neutron scattering enabled the measurements of the frequencies of lattice waves for any chosen wave vector, $\omega(\mathbf{k})$, for a range of elemental solids and simple ionic materials, with modelling of the results including fitting of values of model parameters. One key insight that arose from this work was to understand the role of atomic polarisability in determining frequencies, which in turn allowed a new understanding of dielectric properties (see discussion of the shell model in Section 6). Moreover, very soon after this work came a new understanding of displacive phase transitions - phase transitions where a loss of symmetry is achieved through small displacements of the mean positions of atoms from their positions in the higher-symmetry structure - in terms of lattice dynamics and the soft-mode model [10]. ${ }^{4}$

\footnotetext{
${ }^{3}$ It is interesting to note that citations of, say, the two Born-von Kàrmàn papers [6, 7], are more prevalent in the past few years than in the several decades since their publications.

4 The soft-mode model is not discussed further here. Briefly it states that any second-order displacive phase transition will be accompanied by a vibration whose frequency falls to zero at the transition temperature, called the soft mode, and which corresponds to the loss of resistance against the corresponding atomic displacements and a divergence of the corresponding susceptibility. Soft modes are also associated with first-order displacive phase transitions, but the discontinuous nature of firstorder phase transitions means that the frequency of the soft mode doesn't fall to zero.
} 


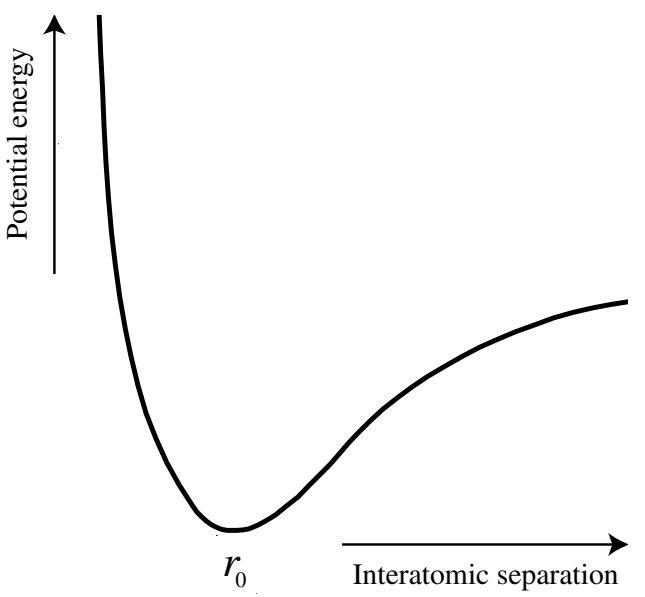

Figure 1. Traditional potential energy curve for two atoms, showing a minimum at a separation of $r_{0}$ that might correspond to the bond length, the steep rise for shorter distances that reflects the repulsion due to overlap of electron density of the two atoms, and the more gentle rise towards zero for larger separations reflecting the attractive interaction. This plot is however somewhat of a simplification of the situation within a crystal, because typically the atoms within a crystal are held in place by a large number of interactions, including the long-range Coulomb interaction, and the position of the minimum of any pair of atoms may not reflect actual equilibrium contact distances.

More recently we have seen a number of key developments in the study of lattice dynamics. In terms of experiments, we are seeing a new generation of instruments at neutron scattering facilities, particularly with the ability to collect data over wide ranges of scattering vector and energy simultaneously. The new instrumentation is matched by software for simulating the outputs of experiments, coupled with new capabilities to calculate $\omega(\mathbf{k})$ from quantum mechanical simulations. These capabilities coincide with the emergence of investigations concerned with new phenomena such as negative thermal expansion, which directly need calculations and measurements of lattice dynamics for a clear understanding (see Section 6.5 for example). All these developments have led to renewed interest in lattice dynamics.

\subsection{The harmonic approximation}

The key approximation in the theory of lattice dynamics is the harmonic approximation. This is illustrated by considering the potential energy between two atoms, as shown in Figure 1. We can write the energy as a Taylor expansion around the minimum point $r_{0}$ :

$$
E(r)=E_{0}+\left.\frac{1}{2} \frac{\partial^{2} E}{\partial r^{2}}\right|_{r_{0}}\left(r-r_{0}\right)^{2}+\left.\frac{1}{3 !} \frac{\partial^{3} E}{\partial r^{3}}\right|_{r_{0}}\left(r-r_{0}\right)^{3}+\left.\frac{1}{4 !} \frac{\partial^{4} E}{\partial r^{4}}\right|_{r_{0}}\left(r-r_{0}\right)^{4}+\cdots
$$

where the derivatives are performed at $r=r_{0} .{ }^{5}$ The harmonic approximation consists of neglecting all terms of power higher than 2.

One might think that the harmonic approximation is both trivial and drastic, but it is actually very powerful. On one hand, it is effectively the only model for lattice dynamics that has an exact solution. On the other hand, it gives us many features that survive addition of higher-order terms. These include the link between vibrational frequencies, wave vector and interatomic forces, and applications in areas

\footnotetext{
${ }^{5}$ In this example the linear term is zero because the definition of equilibrium is that $\partial E / \partial r=$ at the equilibrium distance $r_{0}$.
} 


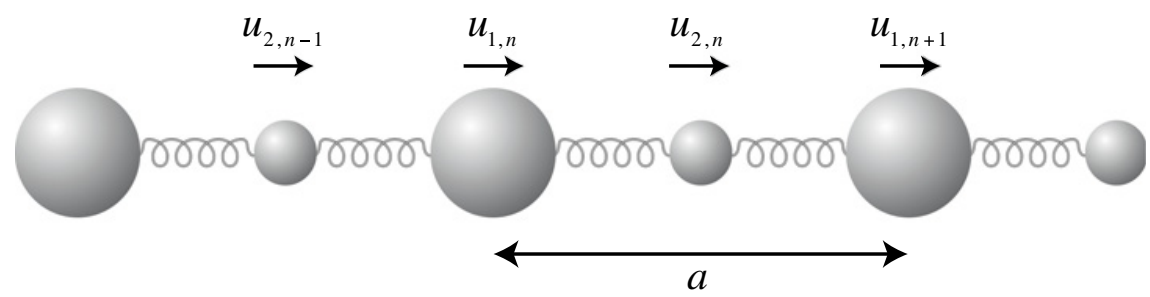

Figure 2. Simple diatomic chain model, with atoms of different mass connected by harmonic forces that are of equal strength between all nearest-neighbour atom pairs. The unit cell length is denoted by $a$, atom a vector of the form $u_{i, n}$ denote the displacement of atom of label $i$ in unit cell of label $n$ [2].

such as the thermodynamic properties of materials. Moreover, the harmonic model is easily adapted to incorporate quantum mechanics.

Thus there is considerable merit in starting with the harmonic approximation, and then attempting to modify the picture to account for higher-order anharmonic terms as appropriate. Applications that are not explained by the harmonic model include properties such as thermal expansion and thermal conductivity, and behaviour such as phase transitions. Experience has shown that for many of these applications this approach works well. For example, in the study of thermal expansion it is possible to retain the harmonic approximation but allow force constants to change with an expansion of the lattice.

\section{THEORY OF HARMONIC LATTICE DYNAMICS}

\subsection{Starting model: The diatomic chain}

Most textbooks begin with a model that consists of one atom in the unit cell, which is typically then explored in a single dimension and subsequently generalised to three dimensions visually. Here we will skip past this approach and introduce instead a one-dimensional model of a crystal containing two atoms in the unit cell, Figure 2. By starting with this model we quickly position ourselves to generalise the formalism to more complex three-dimensional materials.

The total energy of this model is written in terms of the displacements of atoms 1 and $2, u_{1, n}$ and $u_{2, n}$ respectively, as defined in Figure 2:

$$
\begin{aligned}
E & =\frac{1}{2} J \sum_{n}\left(u_{1, n}-u_{2, n}\right)^{2}+\frac{1}{2} J \sum_{n}\left(u_{2, n}-u_{1, n+1}\right)^{2} \\
& =J \sum_{n}\left(u_{1, n}^{2}+u_{2, n}^{2}\right)-J \sum_{n}\left(u_{1, n} u_{2, n}+u_{2, n} u_{1, n+1}\right)
\end{aligned}
$$

The first representation reflects the image of the model in terms of bonds as simple springs, with each term corresponding to the energy associate with stretching or compressing one of the springs. The second representation is a Taylor expansion of the total energy, which in general terms can be written as

$$
E=\frac{1}{2} \sum_{i, j} u_{i} \frac{\partial E}{\partial u_{i} \partial u_{j}} u_{j}
$$

By comparing the two preceding equations, it can be seen that the parameter $J$ is equal to the derivative of the total energy:

$$
J=\frac{\partial^{2} E}{\partial u_{1, n} \partial u_{2, n}}
$$




\subsection{Travelling waves}

We next consider the equations of the waves travelling through crystals. In the general case, a wave of wave vector $\mathbf{k}$ and angular frequency $\omega$ travelling through a crystal will displace an atom labelled $j$ at nominal position $\mathbf{r}_{j}$ by

$$
\mathbf{u}_{j}\left(\mathbf{r}_{j}, t\right)=\tilde{\mathbf{u}}_{j} \exp \left(i\left(\mathbf{k} \cdot \mathbf{r}_{j}-\omega t\right)\right)
$$

where $\tilde{\mathbf{u}}_{j}$ represents both the amplitude of the wave and its specific effect on atom $j$, and may be a complex number (this is discussed in more detail in following sections). We remark here that the definition of the position $\mathbf{r}_{j}$ is treated in two ways in the scientific literature. It can be taken to represent the actual position of the atom, or else it can be taken as the origin of the unit cell containing the atom. It actually doesn't matter, because the difference is merely a phase factor, which can be incorporated into the complex amplitude $\tilde{\mathbf{u}}_{j}$.

If we consider a single wave travelling through our one-dimensional mode with a particular value of $\mathbf{k}$ and $\omega$, it will displace the two atoms by

$$
\begin{aligned}
& u_{1, n}(t)=\tilde{u}_{1} \exp (i(k n a-\omega t)) \\
& u_{2, n}(t)=\tilde{u}_{2} \exp (i(k n a-\omega t))
\end{aligned}
$$

where $\tilde{u}_{1}$ and $\tilde{u}_{2}$ are the relative amplitudes of motion of the two atoms. In this case we have treated the vector $\mathbf{r}_{j}$ for both atoms as the origin of the unit cell, $r_{1}=r_{2}=n a$, rather than as the actual positions of the atoms, $n a$ and $(n+1 / 2) a$ respectively. Thus the amplitude $\tilde{u}_{2}$ will contain the phase factor $\exp (i k a / 2)$. At this point we do not know the relationship between $\tilde{u}_{1}$ and $\tilde{u}_{2}$, nor will be able to think about their absolute values until we introduce thermodynamics into the picture.

\subsection{Equations of motion}

Our starting point is to consider the energy of the two atoms in the unit cell labelled $n$ through its interaction with their two nearest neighbours:

$$
\begin{aligned}
& E_{1, n}=\frac{1}{2} J\left(u_{1, n}-u_{2, n}\right)^{2}+\frac{1}{2} J\left(u_{1, n}-u_{2, n-1}\right)^{2} \\
& E_{2, n}=\frac{1}{2} J\left(u_{2, n}-u_{1, n}\right)^{2}+\frac{1}{2} J\left(u_{2, n}-u_{1, n+1}\right)^{2}
\end{aligned}
$$

The key equation we will be dealing with is simply Newton's equation, force $=$ mass $\times$ acceleration. Thus we start by computing the force acting on each atom, as given by the derivative of the energy with respect to displacement:

$$
\begin{aligned}
f_{1, n}=-\frac{\partial E_{1, n}}{\partial u_{1, n}} & =-J\left(u_{1, n}-u_{2, n}\right)-J\left(u_{1, n}-u_{2, n-1}\right) \\
& =-J\left(2 u_{1, n}-u_{2, n}-u_{2, n-1}\right) \\
f_{2, n}=-\frac{\partial E_{2, n}}{\partial u_{2, n}} & =-J\left(u_{2, n}-u_{1, n}\right)-J\left(u_{2, n}-u_{1, n+1}\right) \\
& =-J\left(2 u_{2, n}-u_{1, n}-u_{1, n+1}\right)
\end{aligned}
$$

We next need to consider the acceleration of each atom, which is given as the second time derivative of the atomic displacement:

$$
\begin{aligned}
& \ddot{u}_{1, n}(t)=-\omega^{2} \tilde{u}_{1} \exp i(k n a-\omega t)=-\omega^{2} u_{1, n}(t) \\
& \ddot{u}_{2, n}(t)=-\omega^{2} \tilde{u}_{2} \exp i(k n a-\omega t)=-\omega^{2} u_{2, n}(t)
\end{aligned}
$$


Newton's equation now links equations (2.9) and (2.10) to equations (2.11) and (2.12) respectively to give:

$$
\begin{aligned}
& m_{1} \ddot{u}_{1, n}(t)=-m_{1} \omega^{2} u_{1, n}(t)=-J\left(2 u_{1, n}(t)-u_{2, n}(t)-u_{2, n-1}(t)\right) \\
& m_{2} \ddot{u}_{2, n}(t)=-m_{2} \omega^{2} u_{2, n}(t)=-J\left(2 u_{2, n}(t)-u_{1, n}(t)-u_{1, n+1}(t)\right)
\end{aligned}
$$

Before we proceed, we note that we can write

$$
u_{j, n \pm 1}(t)=u_{j, n} \exp ( \pm i k n a)
$$

Thus we can rewrite equations (2.13) and (2.14), removing the minus signs from both sides, as

$$
\begin{aligned}
& m_{1} \omega^{2} u_{1, n}(t)=J\left(2 u_{1, n}(t)-u_{2, n}(t)-u_{2, n}(t) \exp (-i k a)\right) \\
& m_{2} \omega^{2} u_{2, n}(t)=J\left(2 u_{2, n}(t)-u_{1, n}(t)-u_{1, n}(t) \exp (+i k a)\right)
\end{aligned}
$$

Since both $u_{1, n}(t)$ and $u_{2, n}(t)$ have the same complex exponential function, we can divide this out from both sides of equations (2.16) and (2.17) to yield

$$
\begin{aligned}
& m_{1} \omega^{2} \tilde{u}_{1}=J\left(2 \tilde{u}_{1}-\tilde{u}_{2}-\tilde{u}_{2} \exp (-i k a)\right) \\
& m_{2} \omega^{2} \tilde{u}_{2}=J\left(2 \tilde{u}_{2}-\tilde{u}_{1}-\tilde{u}_{1} \exp (+i k a)\right)
\end{aligned}
$$

It will prove useful going forward to normalise by the atomic masses. We write

$$
e_{1}=m_{1}^{1 / 2} \tilde{u}_{1} ; \quad e_{2}=m_{2}^{1 / 2} \tilde{u}_{2}
$$

Equations (2.18) and (2.19) are thus re-written as

$$
\begin{aligned}
& \omega^{2} e_{1}=J\left(2 e_{1} / m_{1}-e_{2}(1+\exp (-i k a)) / \sqrt{m_{1} m_{2}}\right) \\
& \omega^{2} e_{2}=J\left(2 e_{2} / m_{2}-e_{1}(1+\exp (+i k a)) / \sqrt{m_{1} m_{2}}\right)
\end{aligned}
$$

By inspection, it can be seen that equations (2.21) and (2.22) can be combined into a matrix equation:

$$
\omega^{2}\left(\begin{array}{l}
e_{1} \\
e_{2}
\end{array}\right)=\mathbf{D}(k) \cdot\left(\begin{array}{l}
e_{1} \\
e_{2}
\end{array}\right)
$$

where

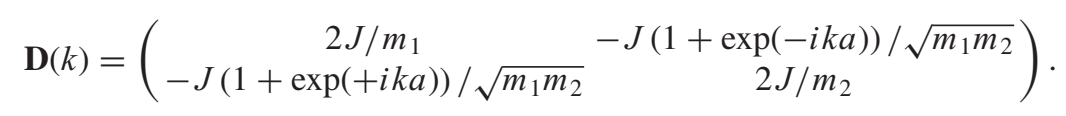

\subsection{Solutions}

Before we rush ahead to discuss the solutions to these equations in detail, there are several points to make here. First, this is a simple eigenvalue/eigenvector equation, with the $\omega^{2}$ values being obtained as the eigenvalues of the matrix $\mathbf{D}(k)$. This will yield two solutions for $\omega^{2}$, which means that our dynamical equations have given two normal modes. Second, the matrix $\mathbf{D}(k)$ has the property that it is equal to the transpose of its complex conjugate. Matrices with this property are called Hermitian, and an important property of Hermitian matrices is that their eigenvalues are real. This means that the values of $\omega^{2}$ obtained as the solutions of the model are real quantities, although they can be negative as well as positive. $^{6}$

\footnotetext{
6 A negative value of $\omega^{2}$ means that the value of $\omega$ will be imaginary; the physical interpretation is that the potential energy surface is curved downwards with respect to the displacements of atoms in the normal mode rather than the expected upwards curvature, which means that that the crystal is actually unstable with respect to the set of displacements. This interpretation forms the basis of the soft-mode model for displacive phase transitions.
} 
Before we plot $\omega^{2}(k)$ as solutions of $\mathbf{D}(k)$ for all values of $k$, let us consider the case of very small values of $k$. We write $\mathbf{D}(k \rightarrow 0)$ as

$$
\mathbf{D}(k \rightarrow 0)=\left(\begin{array}{cc}
2 J / m_{1} & -J(2-i k a) / \sqrt{m_{1} m_{2}} \\
-J(2+i k a) / \sqrt{m_{1} m_{2}} & 2 J / m_{2}
\end{array}\right)
$$

It's eigenvalues are obtained as the solution of the equation

$$
\left|D(k)-\left(\begin{array}{cc}
\omega_{1}^{2}(k) & 0 \\
0 & \omega_{2}^{2}(k)
\end{array}\right)\right|=0
$$

This procedure is frequently called the diagonalisation of the matrix $\mathbf{D}(k)$, because it results in the diagonal matrix whose elements are $\omega_{1}^{2}$ and $\omega_{2}^{2}$. The solutions are obtained as the roots of the equation

$$
\omega^{4}-\frac{2 J\left(m_{1}+m_{2}\right)}{m_{1} m_{2}} \omega^{2}-\frac{J^{2} k^{2} a^{2}}{m_{1} m_{2}}=0
$$

yielding

$$
\omega_{1}^{2}(k)=\frac{J^{2} a^{2}}{2\left(m_{1}+m_{2}\right)} k^{2} ; \quad \omega_{2}^{2}(k)=2 J\left(\frac{1}{m_{1}}+\frac{1}{m_{2}}\right)-O\left(k^{2}\right)
$$

The solution $\omega_{1}^{2}(k)$ has the form $\omega_{1} \propto k$, which corresponds to a sound wave with velocity $v=$ $\omega / k=J a / \sqrt{2\left(m_{1}+m_{2}\right)}$. The second solution has a non-zero value at $k \sim 0$, and also has zero gradient, $\left(\partial \omega_{2} / \partial k\right)_{k=0}=0$. This gradient corresponds to the group velocity - the velocity of energy propagation - and its zero value is characteristic of a standing wave. Given that at $k=0$ every unit cell behaves the same, we expect all solution for $k=0$ other than the sound waves to be standing waves.

We now consider the eigenvectors corresponding to these two solutions. The results are

$$
\begin{array}{ll}
\text { Solution 1: } & m_{1}^{-1 / 2} e_{1}=m_{2}^{-1 / 2} e_{2} \\
\text { Solution 2: } & m_{1}^{1 / 2} e_{1}=-m_{2}^{1 / 2} e_{2}
\end{array}
$$

The eigenvectors of the first solution are consistent with the suggestion above that this wave is a sound wave, namely where neighbouring atoms move in phase with each other with the same amplitude. The eigenvectors of the second solution correspond to neighbouring atoms of different types moving out of phase, with the mass normalisations implying that the centre of mass of the unit cell is not displaced in the wave. These two waves are illustrated in Figure 3. Conventionally the sound wave is called an acoustic mode - for obvious reasons - and the second solution is called an optic mode. The origin of this name comes from the fact that if the two atoms are of opposite charge, the atomic motions represent the displacements that would be caused by a sinusoidally-varying electric field, namely an electromagnetic wave. For many crystals, the frequency of this wave is just short of the frequencies of visible light (typically in the infrared region).

Now we consider a second special case, namely $k=\pi / a$, corresponding to the wavelength of the wave equal to twice the unit cell repeat distance. We can now write

$$
\mathbf{D}(k=\pi / a)=\left(\begin{array}{cc}
2 J / m_{1} & 0 \\
0 & 2 J / m_{2}
\end{array}\right)
$$

The two eigenvalues obtained from diagonalisation of $\mathbf{D}(k=\pi / a)$ are

$$
\omega_{1}^{2}=2 J / m_{1} ; \quad \omega_{2}^{2}=2 J / m_{2}
$$

with eigenvectors for the two solutions

$$
\begin{array}{lll}
\text { Solution 1: } & e_{1}=1 ; & e_{2}=0 \\
\text { Solution 2: } & e_{1}=0 ; & e_{2}=1
\end{array}
$$



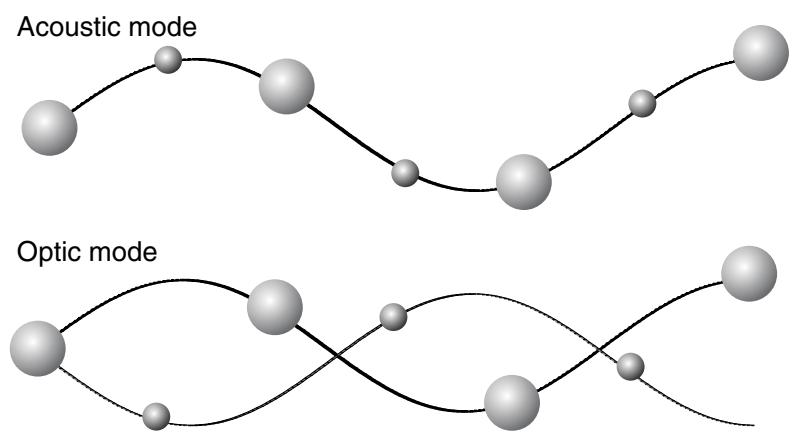

Figure 3. Representation of the difference between acoustic and optic modes in the limit of wave vector $k \rightarrow 0$ for the model diatomic chain. The atomic motions of the two types of atoms are in-phase for the acoustic mode, and out-of-phase for the optic mode [2].

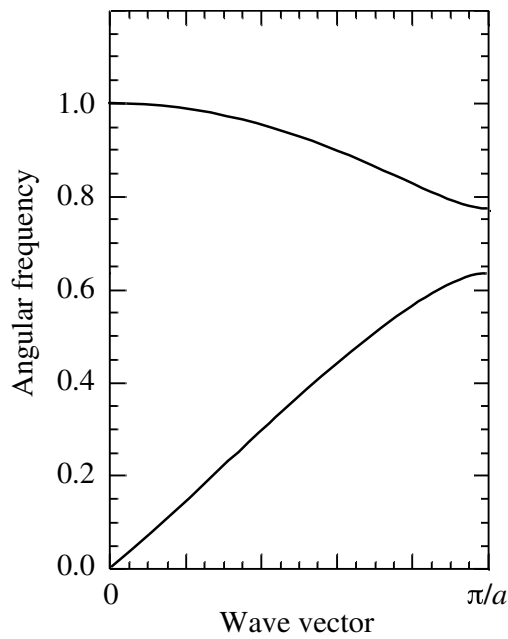

Figure 4. Dispersion curve of the diatomic chain model shown in Figure 2 [2].

These solutions both correspond to one atom remaining at rest in each unit cell, and with $k=\pi / a$ the other atom will move in opposite directions in neighbouring unit cells. Note that in this case, the differentiation between acoustic and optic modes has now vanished. The distinction between in-phase and out-of-phase motions only arises in the limit $k \rightarrow 0$.

The complete set of solutions for $\omega(\mathbf{k})$ for all values of $k$ is shown in Figure 4. These are displayed as two continuous curves, one for the acoustic mode (which becomes the sound wave with $\omega \propto k$ as $k \rightarrow 0$ ) and the other for the optic mode.

We complete this description by noting three features of the dispersion curves shown in figure 4. First, both solutions at $k=\pi / a$ have zero group velocity, that is $\partial \omega / \partial k=0$. At this wave vector, both waves are standing waves, they correspond to the motions of atoms in neighbouring unit cells being exactly opposite to each other. The second point is that the solutions for any $\mathbf{k}$ are invariant with respect to changing the sign of $\mathbf{k}$. The third point is that the solutions are also invariant when adding any reciprocal lattice vector, which in our simple model would be given by $\pm 2 \pi n / a$, where $n$ is any integer. 


\subsection{Generalisation of the model}

The simple model is easily generalised. First we consider more distant neighbours. To make this easier, we combine and rewrite equations (2.7) and (2.8) as

$$
E=\frac{1}{4} \sum_{n, n^{\prime}} \sum_{j, j^{\prime}} \phi_{n, n^{\prime}}^{j, j^{\prime}}\left(u_{j, n}-u_{j^{\prime}, n^{\prime}}\right)^{2}=\frac{1}{2} \sum_{n, n^{\prime}} \sum_{j, j^{\prime}} u_{j, n} \Phi_{n, n^{\prime}}^{j, j^{\prime}} u_{j^{\prime}, n^{\prime}}
$$

where $\phi_{j, j^{\prime}}^{n, n^{\prime}}$ is the differential of an individual bond energy with respect to the displacements of the atoms within the bond, $\Phi_{j, j^{\prime}}^{n, n^{\prime}}$ is the differential of the overall energy with respect to the atomic displacements, and the factors of $1 / 4$ instead of $1 / 2$ arise because we need to account for the fact that the equation as written involves counting every interatomic distance twice. The labels $n$ and $n^{\prime}$ denote unit cells, and the labels $j$ and $j^{\prime}$ denote atoms in the unit cell. In our initial model, $j$ and $j^{\prime}$ had values 1 or 2 , and we restricted the set of $n$ and $n^{\prime}$ to same and nearest-neighbour unit cells. This generalisation now allows more than two atoms in the unit cell, and allows interactions between atoms to span distances larger than nearest neighbours. Close inspection of equation 2.35 shows that

$$
\Phi_{n, n^{\prime}}^{j, j^{\prime}}=-\phi_{n, n^{\prime}}^{j, j^{\prime}}+\sum_{j^{\prime}, n^{\prime}} \delta_{j, j^{\prime}} \delta_{n, n^{\prime}} \phi_{n, n^{\prime}}^{j, j^{\prime}}
$$

We proceed by writing the equation of motion for any atom in the unit cell as

$$
u_{j, n}(t)=\tilde{u}_{j} \exp (i(k n a-\omega t))
$$

Newton's equations for this generalised model for the atoms in unit cell labelled $n$ are now given as

$$
\omega^{2} e_{j}=\sum_{j^{\prime}, n^{\prime}} \frac{1}{\sqrt{m_{j} m_{j^{\prime}}}} \Phi_{n, n^{\prime}}^{j, j^{\prime}} \exp \left(i k\left(n^{\prime}-n\right) a\right) e_{j^{\prime}}
$$

We can expand this in the form ${ }^{7}$

$$
\rightarrow \omega^{2} \mathbf{e}=\mathbf{D}(\mathbf{k}) \cdot \mathbf{e} \quad \Rightarrow \quad \omega^{2}=\mathbf{e}^{T} \cdot \mathbf{D}(\mathbf{k}) \cdot \mathbf{e}
$$

where

$$
\mathbf{e}=\left(\begin{array}{c}
\vdots \\
e_{j} \\
\vdots
\end{array}\right)
$$

and

$$
-\quad D_{j, j^{\prime}}(\mathbf{k})=\frac{1}{\sqrt{m_{j} m_{j^{\prime}}}} \sum_{n^{\prime}} \Phi_{0, n^{\prime}}^{j, j^{\prime}} \exp \left(i \mathbf{k} \cdot\left(\mathbf{r}_{j, 0}-\mathbf{r}_{j^{\prime}, n^{\prime}}\right)\right)
$$

and where we have generalised to three dimensions in our description of the wave vector and atomic positions. Clearly in making the generalisation to three dimensions the matrix $\Phi$ also needs to be expanded to include derivatives of the energy by the vector components of the displacements, but the book-keeping becomes sufficiently complex (we need another pair of subscripts denoting $x, y$ and $z$ vector components for the elements of matrix $\Phi)$ that for the purposes here it is best left to your imagination.

Equations (2.39)-(2.41) represent diagonalisation of the dynamical matrix $\mathbf{D}(\mathbf{k})$, with the matrix of solutions $\omega^{2}$ representing the eigenvalues of $\mathbf{D}(\mathbf{k})$ and the matrix of e representing the eigenvectors.

\footnotetext{
${ }^{7}$ Here we use the symbol $>$ to denote a key equation here and onwards in this article.
} 


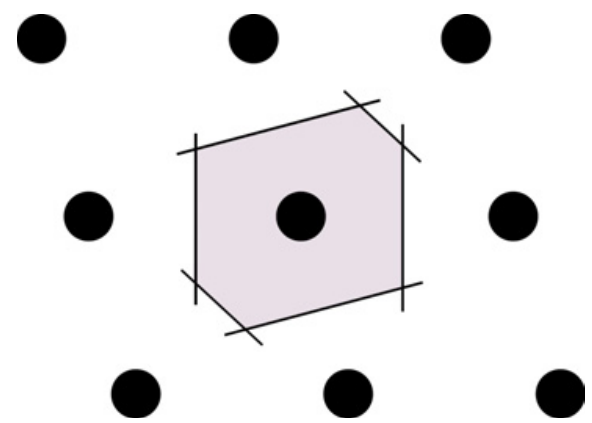

Figure 5. Schematic representation of the Brillouin zone in two dimensional reciprocal space. The boundaries bisect and are normal to the vectors from the origin to the neighbouring reciprocal lattice points [2].

The task that we now move on to discuss is how to calculate the components of $\mathbf{D}(\mathbf{k})$ from models of the interatomic potentials.

\subsection{Brillouin zone}

The wave equations introduced in equations (2.5) and (2.6) have one important property. If we add a reciprocal lattice vector, $G=2 \pi h / a$ (where $h$ is an integer) to a value of $k$ we find that

$$
\exp (i(G+k) n a)=\exp (i G n a) \times \exp (i k n a)=\exp (i n h 2 \pi) \times \exp (i k n a)=\exp (i k n a)
$$

Thus we find that waves of wave vector $k$ and $k+G$ are identical in terms of their impact on the atoms. ${ }^{8}$ This result is easily generalise to three dimensions.

This being the case, we only need to consider the set of wave vectors that are not related to each other by addition of a reciprocal lattice vector $\mathbf{G}$. This set will be contained within the space around the origin of reciprocal space of volume equal to the reciprocal unit cell. It is convenient to work with a space-filling volume that is equivalent in size to the reciprocal unit cell but with boundaries that bisect the vectors between the origin and neighbouring reciprocal lattice points rather than linking reciprocal lattice points. This is illustrated in two dimensions in Figure 5. The boundaries of the Brillouin zone have a particular significance in the nature of the dispersion curves, in that the zone boundaries usually have $\partial \omega / \partial k=0 .^{9}$

\section{NORMAL MODE COORDINATES AND VIBRATIONAL AMPLITUDES}

\subsection{Definition of the normal mode coordinates}

Up to this point we have said nothing about the amplitudes of vibrations save for noting that the e eigenvector matrix contains information about relative atomic displacements. We denote a particular eigenvector as $\mathbf{e}_{\lambda}$, where $\lambda$ labels the eigenvector (or branch in the dispersion curve diagram), with corresponding eigenvalue $\omega_{\lambda}^{2}$. We will also take it to be the case that eigenvectors are normalised and orthogonal (the latter condition is determined by the mathematics of eigenvectors; the normalisation condition is arbitrary but reasonable):

$$
\mathbf{e}_{\lambda}^{\mathrm{T}} \cdot \mathbf{e}_{\lambda}=1 ; \quad \mathbf{e}_{\lambda^{\prime}}^{\mathrm{T}} \cdot \mathbf{e}_{\lambda}=\delta_{\lambda^{\prime}, \lambda}
$$

\footnotetext{
8 The two waves are not the same in the space between the atoms, but that is only empty space and the difference has no meaning. 9 The exception is when two modes are degenerate at the zone boundary but of different frequencies away from the zone boundary, in which case the values of $\partial \omega / \partial k$ for the two modes sum to zero.
} 
We can now write the displacement of a single atom, labelled $j$ in the unit cell of label $\ell$ in terms of the mode eigenvector:

$$
-u_{j \ell}(t)=\frac{1}{\sqrt{N m_{j}}} \sum_{\mathbf{k}, \lambda} \mathbf{e}_{\mathbf{k}, \lambda} \exp \left(i \mathbf{k} \cdot \mathbf{r}_{j \ell}\right) Q(\mathbf{k}, \lambda, t)
$$

where we have now associated each mode eigenvector with a wave vector $\mathbf{k}$, and introduced a new complex quantity $Q(\mathbf{k}, \lambda, t)$ that absorbs the time dependence and the actual amplitude. This new quantity is called the normal mode coordinate. Equation (3.2) expresses the Fourier relationship between atomic displacements in real space and the normal mode coordinates in reciprocal space. The factor of $1 / \sqrt{m_{j}}$ reflects the fact that the mode eigenvector contains a factor of $\sqrt{m_{j}}$, and the factor of $1 / \sqrt{N}$ will be seen to be convenient when we sum over all atoms.

Equation (3.2) can be adapted for the atomic velocity:

$$
\dot{u}_{j \ell}(t)=\frac{1}{\sqrt{N m_{j}}} \sum_{\mathbf{k}, \lambda} \mathbf{e}_{\mathbf{k}, \lambda} \exp \left(i \mathbf{k} \cdot \mathbf{r}_{j \ell}\right) \dot{Q}(\mathbf{k}, \lambda, t)
$$

We note that since $Q(\mathbf{k}, \lambda, t)$ absorbs the time dependence, it will follow that

$$
\dot{Q}(\mathbf{k}, \lambda, t)=-i \omega_{\mathbf{k}, \lambda} Q(\mathbf{k}, \lambda, t)
$$

and thus we can rewrite equation (3.3) as

$$
\dot{u}_{j \ell}(t)=\frac{-i}{\sqrt{N m_{j}}} \sum_{\mathbf{k}, \lambda} \omega_{\mathbf{k}, \lambda} \mathbf{e}_{\mathbf{k}, \lambda} \exp \left(i \mathbf{k} \cdot \mathbf{r}_{j \ell}\right) Q(\mathbf{k}, \lambda, t)
$$

\subsection{Energy of the crystal in terms of the normal mode coordinates}

The prior definitions are convenient in going forward to compute the total kinetic energy of the crystal in terms of its atomic vibrations. The final result, derived in Appendix A, is

$$
\frac{1}{2} \sum_{j, \ell} m_{j}\left|\dot{\mathbf{u}}_{j \ell}\right|^{2}=\frac{1}{2} \sum_{\mathbf{k}, \lambda} \omega_{\mathbf{k}, \lambda}^{2}|Q(\mathbf{k}, \lambda)|^{2}
$$

Similarly, the harmonic potential energy of the crystal can be written as

$$
\frac{1}{2} \sum_{\substack{j, j^{\prime} \\ \ell, \ell^{\prime}}} \mathbf{u}_{j \ell}^{T} \cdot \Phi_{\ell, \ell^{\prime}}^{j, j^{\prime}} \cdot \mathbf{u}_{j^{\prime} \ell^{\prime}}=\frac{1}{2} \sum_{\mathbf{k}, \lambda} \omega_{\mathbf{k}, \lambda}^{2}|Q(\mathbf{k}, \lambda)|^{2}
$$

which is derived in Appendix A. Thus the total vibrational energy - kinetic energy plus potential energy - is written as

$$
>\frac{1}{2} \sum_{j, \ell} m_{j}\left|\dot{\mathbf{u}}_{j \ell}\right|^{2}+\frac{1}{2} \sum_{\substack{j, j^{\prime} \\ \ell, \ell^{\prime}}} \mathbf{u}_{j \ell}^{T} \cdot \Phi_{\ell, \ell^{\prime}}^{j, j^{\prime}} \cdot \mathbf{u}_{j^{\prime} \ell^{\prime}}=\sum_{\mathbf{k}, \lambda} \omega_{\mathbf{k}, \lambda}^{2}|Q(\mathbf{k}, \lambda)|^{2} .
$$

\subsection{Quantisation of the vibrational energy: Phonons}

To make further progress we need to understand that the energy of a harmonic oscillation is quantised in units of $\hbar \omega$. We are most familiar with these quanta being applied to light, where they are called photons. However, this quantisation applies to all harmonic vibrations, and a single wave of atomic oscillations is similarly quantised; the quantum in this case is called a phonon. 
The energy of a single oscillation that is quantised can be written as the number of phonons excited, $n$, plus a constant value:

$$
-E_{n}=\left(n+\frac{1}{2}\right) \hbar \omega
$$

the additional constant value of $\hbar \omega / 2$ is called the zero point energy, and reflects the fact that in quantum mechanics a harmonic oscillator can never be at rest. Thus we can write equation (3.8) as

$$
E=\sum_{\mathbf{k}, \lambda} \omega_{\mathbf{k}, \lambda}^{2}|Q(\mathbf{k}, \lambda)|^{2}=\sum_{\mathbf{k}, \lambda}\left(n_{\mathbf{k}, \lambda}+\frac{1}{2}\right) \hbar \omega_{\mathbf{k}, \lambda}
$$

We have effectively switched the question from the wanting to know about the amplitude of the normal mode to one of knowing the value of $n_{\mathbf{k}, \lambda}$. In practice it is not the instantaneous value of $n_{\mathbf{k}, \lambda}$ that we need, but its average value at a particular temperature. It turns out that the average value of $n_{\mathbf{k}, \lambda}$ only depends on $\mathbf{k}$ and $\lambda$ through the dependence on $\omega_{\mathbf{k}, \lambda}$ :

$$
-\left\langle n\left(\omega_{\mathbf{k}, \lambda}\right)\right\rangle=\frac{1}{\exp \left(\hbar \omega_{\mathbf{k}, \lambda} / k_{\mathrm{B}} T\right)-1}
$$

This is known as the Bose-Einstein equation. Given that the average number of excited phonons depends only on the frequency, and that in a harmonic system its excited waves are independent of each other, we can extract a single normal mode and write

$$
\omega_{\mathbf{k}, \lambda}^{2}\left\langle|Q(\mathbf{k}, \lambda)|^{2}\right\rangle=\left(\left\langle n\left(\omega_{\mathbf{k}, \lambda}\right)\right\rangle+\frac{1}{2}\right) \hbar \omega_{\mathbf{k}, \lambda}
$$

It is useful at this point to note that in the limit $k_{\mathrm{B}} T>\hbar \omega_{\mathbf{k}, \lambda}$, the Bose-Einstein relation tends - actually remarkably quickly - towards the approximate form

$$
\left\langle n\left(\omega_{\mathbf{k}, \lambda}\right)\right\rangle+\frac{1}{2} \rightarrow k_{\mathrm{B}} T / \hbar \omega_{\mathbf{k}, \lambda}
$$

In this case, the total energy of a single wave tends towards the well-known classical value $k_{\mathrm{B}} T$.

\subsection{Crystal Hamiltonian in terms of the normal mode coordinates}

Using the previous analysis, the Hamiltonian of the harmonic crystal, namely the sum of the kinetic and potential energies, is written in the form of

$$
\triangleright \mathcal{H}=\frac{1}{2} \sum_{j, \ell} m_{j}\left|\dot{\mathbf{u}}_{j \ell}\right|^{2}+\frac{1}{2} \sum_{\substack{j, j^{\prime} \\ \ell, \ell^{\prime}}} \mathbf{u}_{j \ell}^{T} \cdot \Phi_{\ell, \ell^{\prime}}^{j, j^{\prime}} \cdot \mathbf{u}_{j^{\prime} \ell^{\prime}}=\sum_{\mathbf{k}, \lambda}|\dot{Q}(\mathbf{k}, \lambda)|^{2}+\sum_{\mathbf{k}, \lambda} \omega_{\mathbf{k}, \lambda}^{2}|Q(\mathbf{k}, \lambda)|^{2}
$$

This is an extremely powerful equation, in part because it is very simple, and in part also because one can imagine extending this for the effects of higher-order anharmonic interactions:

$$
\mathcal{H}=\frac{1}{2} \sum_{\mathbf{k}, \lambda}\left|\dot{Q}_{\mathbf{k}, \lambda}\right|^{2}+\frac{1}{2} \sum_{\mathbf{k}, \lambda} \omega_{\mathbf{k}, \lambda}^{2}\left|Q_{\mathbf{k}, \lambda}\right|^{2}+\sum_{n} \frac{1}{n !} \sum_{\substack{\mathbf{k}_{1} \cdots \mathbf{k}_{n} \\ \lambda_{1} \cdots \lambda_{n}}} \xi_{\lambda_{1} \cdots \lambda_{n}}^{\mathbf{k}_{1} \cdots \mathbf{k}_{n}} Q_{\mathbf{k}_{1}, \lambda_{1}} \ldots Q_{\mathbf{k}_{n}, \lambda_{n}}
$$

It is outside the scope of this paper to explore this further, but for weakly anharmonic crystals it is possible to treat the anharmonic terms as small perturbations of the harmonic Hamiltonian, and to use various approximation schemes to incorporate them into the harmonic terms with renormalised parameters. 


\section{THERMODYNAMICS AND DENSITY OF STATES}

\subsection{Thermodynamic functions}

In the derivation of the Bose-Einstein distribution given in Appendix B, we derive the equation for the partition function of a harmonic oscillator of angular frequency $\omega, \mathcal{Z}$ :

$$
\mathcal{Z}=\frac{1}{1-\exp (-\beta \hbar \omega)}
$$

The free energy is related to $\mathcal{Z}$ via

$$
F=-k_{\mathrm{B}} T \ln \mathcal{Z}
$$

and thus we obtain

$$
-\quad F=k_{\mathrm{B}} T \sum_{\mathbf{k}, \lambda} \ln \left[2 \sinh \left(\hbar \omega_{\mathbf{k}, \lambda} / 2 k_{\mathrm{B}} T\right)\right]
$$

Other thermodynamic quantities, such as the heat capacity, can be obtained by appropriate differentiation of $F$ (e.g. the heat capacity is equal to $-T \partial^{2} F / \partial T^{2}$ ).

\subsection{Density of states}

Given that the thermodynamic functions only depend on the frequency of the normal mode and not directly on its wave vector or mode eigenvector, one way to perform the summations over all modes and wave vectors is to simply generate a list of frequency values for a grid of wave vectors from one's favourite lattice dynamics program. ${ }^{10}$ If the grid is sufficiently fine, it is possible to then generate a histogram of frequency values, and such a histogram is called the density of states, $g(\omega)$. Formally we note that the density of states is defined such that the number of modes with angular frequency in the range $\omega \rightarrow \omega+\mathrm{d} \omega$ is equal to $g(\omega) \mathrm{d} \omega$. Then the summations in the thermodynamic functions can be replaced by appropriate integrals. For example, the energy can be written as

$$
E=\sum_{\mathbf{k}, \lambda}\left(\left\langle n\left(\omega_{\mathbf{k}, \lambda}\right)\right\rangle+\frac{1}{2}\right) \hbar \omega_{\mathbf{k}, \lambda} \equiv \int\left(\langle n(\omega)\rangle+\frac{1}{2}\right) \hbar \omega g(\omega) \mathrm{d} \omega .
$$

From a computational perspective, this is not particularly interesting. However, in the limit of low-frequency, the density of states only contains contributions from the acoustic modes, and in this case it is possible to obtain a mathematical equation for $g(\omega)$. Moreover, for thermodynamic applications, the only modes that will be excited at low temperatures according to the Bose-Einstein equation are the lower-frequency acoustic modes, and an exact expression for $g(\omega)$ for these modes will enable thermodynamic properties to be calculated exactly. We make the (unnecessary but pedagogical) approximation that the frequencies of the acoustic modes follow a simple linear dependence on wave vector, $\omega=c k$, where $c$ is an average sound velocity. Because this is a linear problem, we can compute $g(\omega)$ from the distribution of wave vector values, $g(k)$. Writing the volume of the crystal as $V$, and defined with $N$ unit cells and hence $N$ wave vectors, the number of wave vectors per unit volume of reciprocal space is equal to $V /(2 \pi)^{3}$. Thus in a spherical shell of radius $k$ and thickness $\mathrm{d} k$, the number of wave vectors will be equal to

$$
g(k) \mathrm{d} k=\frac{V}{(2 \pi)^{3}} 4 \pi k^{2} \mathrm{~d} k .
$$

\footnotetext{
10 Some care is needed in setting up this grid. For example, if it includes special points in reciprocal space, these may need to be weighted slightly differently than general points if the griding is performed over the a symmetrically-unique segment of the Brillouin zone. It may sometimes be useful to use a random set of wave vectors rather than wave vectors across a uniform grid.
} 
Substituting for $k=\omega / c$ and $\mathrm{d} k=\mathrm{d} \omega / c$, we obtain

$$
g(\omega)=\frac{3 V}{2 \pi^{2} c^{3}} \omega^{2}
$$

where the factor of 3 accounts for the number of acoustic modes for each wave vector. The relationship $g(\omega) \propto \omega^{2}$ is a general result that is seen in calculations or measurements on any ordered crystalline materials. If one sees departures from this relationship, the system will contain excitations that are not described by simple harmonic travelling waves. Typically this might be found in disordered materials. ${ }^{11}$

Increasingly we are seeing measurements of the density of states using neutron or x-ray scattering ${ }^{12}$ being used as a probe of variations of phonon frequencies with parameters such as temperature, particularly when single crystals for full measurements of dispersion curves are not available.

\subsection{Heat capacity at low temperatures}

With some manipulation, it can be shown that equation 4.4 for the energy in the limit of low temperature for the form of $g(\omega)$ given by equation 4.6 can be solved to give

$$
E=\frac{V \pi^{2}\left(k_{\mathrm{B}} T\right)^{4}}{10(c \hbar)^{3}}
$$

(See, for example, Chapter 9 of reference 2.) It thus follows that the heat capacity has the form

$$
c_{\mathrm{V}}=\frac{2 V \pi^{2} k_{\mathrm{B}}}{5\left(c \hbar / k_{\mathrm{B}}\right)^{3}} T^{3}=N \frac{12 \pi^{4} k_{\mathrm{B}}}{5}\left(\frac{T}{\theta_{\mathrm{D}}}\right)^{3} ; \quad \theta_{\mathrm{D}}=\frac{c \hbar}{k_{\mathrm{B}}}\left(\frac{6 \pi^{2} N}{V}\right)^{1 / 3}
$$

We see that the heat capacity at low temperature will vary as $T^{3}$, a result that has been confirmed for many crystalline materials. We have written the heat capacity in terms of the material constant $\theta_{\mathrm{D}}$, which is known as the Debye temperature.

This result is important in three regards. First, as noted above, it is seen to be obeyed by a large number of crystalline materials, and this analysis enables to understand why. Second, in metals there is an important contribution to the heat capacity from the electrons that varies linearly with temperature at low temperature, and having an expression for the phonon contribution to the heat capacity enables the electronic component to be extracted. Third, some disordered materials - particularly many amorphous materials - are found to have a heat capacity that varies more closely to linearly with temperature than the $T^{3}$ law; with the theoretical support for the $T^{3}$ law we immediately understand that the departure from this law implies the need for a deeper understanding of the thermodynamics of amorphous materials.

\section{EXPERIMENTAL STUDIES}

\subsection{Classical theory of inelastic neutron and $x$-ray scattering}

In this section we discuss the results from experimental studies of lattice dynamics of a number of different systems. Traditionally the key technique has been inelastic neutron scattering. Before we present representative results, we will sketch a classical theory of inelastic scattering, a theory that will apply also to inelastic scattering of x-rays.

We start by considering the process of elastic scattering radiation from an assembly of atoms, where the scattered beam has the same wavelength/energy as the incident beam. ${ }^{13}$ Figure 6 shows the path of

\footnotetext{
11 It is a simple generalisation of this formalism to show that for $d$-dimensional systems $g(\omega) \propto \omega^{d-1}$.

12 Technically the experimental measurements will be weighted by the scattering power of each atoms.

13 Actually we do not need to assume that the wavelength doesn't change through the scattering process, and the following equations can easily be rewritten allowing for a change of wavelength.
} 


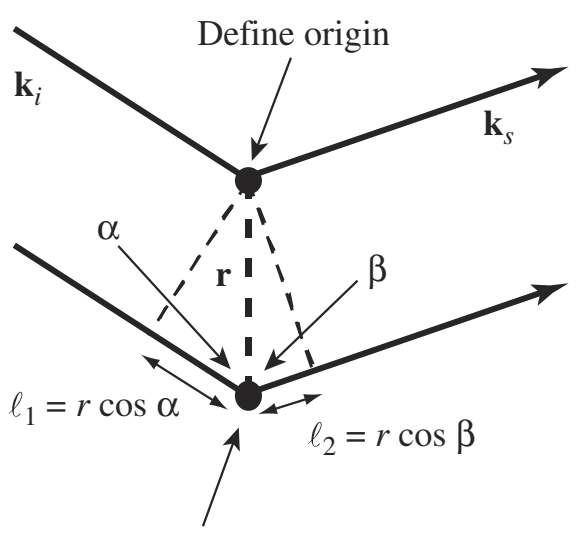

Position $\mathbf{r}$

Figure 6. Schematic diagram of the scattering of a beam of radiation from a point at a position defined by the vector $\mathbf{r}$ from an origin. The vectors $\mathbf{k}_{i}$ and $\mathbf{k}_{s}$ represent the wave vectors of the incident and scattering beams.

the beam of radiation when scattered by a particle at position $\mathbf{r}$ relative to an origin. The figure shows the additional path length $\ell_{1}+\ell_{2}$, and indicates the change in wave vector through the scattering process from $\mathbf{k}_{i}$ to $\mathbf{k}_{s}$ ( $i$ and $s$ denote the incoming and scattered beams). The path length can be expressed in terms of the wave vector as

$$
\ell_{1}=r \cos \alpha_{1}=\frac{\lambda}{2 \pi} \mathbf{k}_{i} \cdot \mathbf{r} ; \quad \ell_{2}=r \cos \alpha_{2}=-\frac{\lambda}{2 \pi} \mathbf{k}_{s} \cdot \mathbf{r}
$$

which translates to a relative change of phase of the scattered beam relative to a beam scattered from the origin as

$$
\frac{2 \pi}{\lambda}\left(\ell_{1}+\ell_{2}\right)=\left(\mathbf{k}_{i}-\mathbf{k}_{s}\right) \cdot \mathbf{r}=\mathbf{Q} \cdot \mathbf{r}
$$

This equation defines the scattering vector $\mathbf{Q}$ as the change in wave vector of the beam through the scattering process:

$$
\mathbf{Q}=\mathbf{k}_{i}-\mathbf{k}_{s}
$$

The total scattering is then the sum over all atoms, weighted by the scattering power of each atom, which in the case of neutron scattering is called the neutron scattering length and denoted by $b_{j}$ :

$$
\rho(\mathbf{Q})=\sum_{j} b_{j} \exp \left(i \mathbf{Q} \cdot \mathbf{r}_{j}\right)
$$

Here $b_{j}$ is assumed - as is the case for neutron scattering - to be independent of $\mathbf{Q}$; in the case of $\mathrm{x}$-ray scattering, the scattering length is replaced by the atomic scattering factor $f_{j}(Q)$, the value of which has a strong dependence on $Q=|\mathbf{Q}|$. It should be noted that the function $\rho(\mathbf{Q})$ is the Fourier transform of the atom density, where the density of a single atom is described by a Dirac delta function, $\rho_{j}(\mathbf{r})=\delta\left(\mathbf{r}-\mathbf{r}_{j}\right)$.

The intensity of the scattered beam is given as

$$
S(\mathbf{Q})=\left\langle|\rho(\mathbf{Q})|^{2}\right\rangle=\sum_{j, j^{\prime}} b_{j} b_{j^{\prime}}\left\langle\exp \left(i \mathbf{Q} \cdot\left(\mathbf{r}_{j}-\mathbf{r}_{j^{\prime}}\right)\right)\right\rangle
$$

where we have introduced the fact that the measurement will involve an average over time. 
We next need to extend this analysis to allow for a phase shift due to scattering from different times. Thus we redefine equation 5.4 to account for the dependence on time:

$$
\rho(\mathbf{Q}, t)=\sum_{j} b_{j} \exp \left(i \mathbf{Q} \cdot \mathbf{r}_{j}(t)\right)
$$

We account for this by the addition of a phase factor $\exp (-i \omega t)$ :

$$
\rho(\mathbf{Q}, t)=\rho(\mathbf{Q}) \exp (-i \omega t)=\sum_{j} b_{j} \exp \left(i \mathbf{Q} \cdot \mathbf{r}_{j}(t)\right) \exp (-i \omega t)
$$

Thus the time-dependence introduces another source of a phase shift, which is represented as $\exp (-i \omega t)$. We thus need to average over all times to obtain the resultant scattering:

$$
\rho(\mathbf{Q}, \omega)=\int \rho(\mathbf{Q}, t) \exp (-i \omega t) \mathrm{d} t
$$

Following our analysis of the scattering from the static structure, we form the intensity of scattering as

$$
\begin{aligned}
S(\mathbf{Q}, \omega) & =\int \rho\left(\mathbf{Q}, t^{\prime}\right) \exp \left(-i \omega t^{\prime}\right) \mathrm{d} t^{\prime} \times \int \rho(-\mathbf{Q}, t) \exp (-i \omega t) \mathrm{d} t \\
& =\iint \rho\left(\mathbf{Q}, t^{\prime}\right) \rho\left(-\mathbf{Q}, t+t^{\prime}\right) \exp (-i \omega t) \mathrm{d} t \mathrm{~d} t^{\prime} \\
& =\int\langle\rho(\mathbf{Q}, 0) \rho(-\mathbf{Q}, t)\rangle \exp (-i \omega t) \mathrm{d} t
\end{aligned}
$$

where the final step is simply performing an average over all time origins. Thus we have the following result:

$$
\checkmark S(\mathbf{Q}, \omega)=\sum_{j, j^{\prime}} b_{j} b_{j^{\prime}} \int\left\langle\exp \left(i \mathbf{Q} \cdot \mathbf{r}_{j}(0)\right) \exp \left(-i \mathbf{Q} \cdot \mathbf{r}_{j^{\prime}}(t)\right)\right\rangle \exp (-i \omega t) \mathrm{d} t
$$

This is the critical equation. What we have given is essentially a classical derivation, but the essential form of the equation remains robust in a quantum mechanical derivation, other than detailed balance requiring the condition

$$
S(\mathbf{Q},-\omega)=S(\mathbf{Q},+\omega) \times \exp \left(\hbar \omega / k_{\mathrm{B}} T\right)
$$

whereas the classical equation is invariant with respect to the sign of $\omega$. We also note that we are assuming that we are dealing with coherent scattering, which is tantamount to assuming that

$$
\sum_{i, j}\left\langle b_{i} b_{j} \exp \left(i \mathbf{Q} \cdot\left(\mathbf{r}_{i}(t)-\mathbf{r}_{j}(0)\right)\right)\right\rangle=\sum_{i, j} \bar{b}_{i} \bar{b}_{j}\left\langle\exp \left(i \mathbf{Q} \cdot\left(\mathbf{r}_{i}(t)-\mathbf{r}_{j}(0)\right)\right)\right\rangle
$$

That is, we are assuming that all atoms of one type scattering radiation in exactly the same way - this is the coherent approximation. Whilst this sounds plausible, there are two cases where this might not be valid. One case is where we have different isotopes that scatter neutrons with a different value of the scattering length $b$. The other case is where there is a dependence on the relative orientation of the spins of the neutron and nucleus. In practice, the coherent approximation fails most strongly - and significantly so - with hydrogen, where the scattering from the two orientations of the spin of the proton are so different that most of the scattering is incoherent. However, for the purposes of this paper, we will focus wholly on coherent scattering processes. 


\subsection{Scattering from phonons}

In the following discussion, it is helpful to define the intermediate scattering function as the time Fourier transform of $S(\mathbf{Q}, \omega)$ :

$$
S(\mathbf{Q}, \omega)=\int F(\mathbf{Q}, t) \exp (-i \omega t) d t
$$

Here we now focus on representing the instantaneous position of an atom in terms of its displacement $\mathbf{u}_{j}(t)$ from its average position $\mathbf{R}_{j}: \mathbf{r}_{j}(t)=\mathbf{R}_{j}+\mathbf{u}_{j}(t)$. Thus we rewrite the intermediate scattering function as

$$
F(\mathbf{Q}, t)=\sum_{i, j} b_{i} b_{j} \exp \left(i \mathbf{Q} \cdot\left(\mathbf{R}_{i}-\mathbf{R}_{j}\right)\right)\left\langle\exp i \mathbf{Q} \cdot\left(\mathbf{u}_{i}(t)-\mathbf{u}_{j}(0)\right)\right\rangle
$$

To evaluate the averages in this equation, we will make use of the following result for harmonic operators:

Thus we can write

$$
\langle\exp (A) \times \exp (B)\rangle=\exp \left(\left\langle(A+B)^{2}\right\rangle / 2\right)
$$

$$
\begin{aligned}
\left\langle\exp \left(i \mathbf{Q} \cdot\left(\mathbf{u}_{i}(t)-\mathbf{u}_{j}(0)\right)\right)\right\rangle= & \exp \left(-\frac{1}{2}\left\langle\left[\mathbf{Q} \cdot \mathbf{u}_{i}(t)\right]^{2}\right\rangle-\frac{1}{2}\left\langle\left[\mathbf{Q} \cdot \mathbf{u}_{j}(0)\right]^{2}\right\rangle\right. \\
& \left.+\left\langle\left[\mathbf{Q} \cdot \mathbf{u}_{i}(t)\right] \times\left[\mathbf{Q} \cdot \mathbf{u}_{j}(0)\right]\right\rangle\right)
\end{aligned}
$$

The first two terms in the exponent are simply the thermal displacement factors (otherwise known as the temperatyre factors), which we will denote as $W_{i}(\mathbf{Q})$ and $W_{j}(\mathbf{Q})$ respectively. The third term is the important one, and it can be represented as a Taylor expansion:

$$
\exp \left\langle\left[\mathbf{Q} \cdot \mathbf{u}_{i}(t)\right] \times\left[\mathbf{Q} \cdot \mathbf{u}_{j}(0)\right]\right\rangle=\sum_{m} \frac{1}{m !}\left\langle\left[\mathbf{Q} \cdot \mathbf{u}_{i}(t)\right] \times\left[\mathbf{Q} \cdot \mathbf{u}_{j}(0)\right]\right\rangle^{m}
$$

The $m=0$ term can be shown to correspond to normal Bragg scattering. ${ }^{14}$ Of more interest here is the $m=1$ term, which corresponds to scattering involving a single phonon as indicated in Figure 7 . The key quantity is

$$
\begin{aligned}
\left\langle\left[\mathbf{Q} \cdot \mathbf{u}_{i}(t)\right]\left[\mathbf{Q} \cdot \mathbf{u}_{j}(0)\right]\right\rangle= & \frac{1}{N \sqrt{m_{i} m_{j}}} \sum_{\substack{\mathbf{k}, \mathbf{k}^{\prime} \\
\lambda, \lambda^{\prime}}}[\mathbf{Q} \cdot \mathbf{e}(i, \mathbf{k}, \lambda)]\left[\mathbf{Q} \cdot \mathbf{e}\left(j, \mathbf{k}^{\prime}, \lambda^{\prime}\right)\right] \\
& \times \exp \left(i \mathbf{k} \cdot \mathbf{R}_{i}\right) \exp \left(i \mathbf{k}^{\prime} \cdot \mathbf{R}_{k}\right)\left\langle Q(\mathbf{k}, \lambda, t) Q\left(\mathbf{k}^{\prime}, \lambda^{\prime}, 0\right)\right\rangle
\end{aligned}
$$

It can be shown that this term resolves to ${ }^{15}$

$$
\begin{aligned}
\left\langle\left[\mathbf{Q} \cdot \mathbf{u}_{i}(t)\right]\left[\mathbf{Q} \cdot \mathbf{u}_{j}(0)\right]\right\rangle= & \frac{1}{N \sqrt{m_{i} m_{j}}} \sum_{\mathbf{k}, \lambda}[\mathbf{Q} \cdot \mathbf{e}(i, \mathbf{k}, \lambda)][\mathbf{Q} \cdot \mathbf{e}(j,-\mathbf{k}, \lambda)] \\
& \times\langle Q(\mathbf{k}, \lambda) Q(-\mathbf{k}, \lambda)\rangle \cos \omega_{\mathbf{k}, \lambda} t
\end{aligned}
$$

The final form of $S(\mathbf{Q}, \omega)$ for the $m=1$ term is derived from this analysis, but when taking quantum mechanics into account it has the modified form

$$
-S_{1}(\mathbf{Q}, \omega)=\sum_{\lambda} \frac{1}{2 \omega_{\mathbf{k}, \lambda}}\left|F_{\lambda}(\mathbf{Q})\right|^{2}\left[(1+n(\omega)) \delta\left(\omega+\omega_{\mathbf{k}, \lambda}\right)+n(\omega) \delta\left(\omega-\omega_{\mathbf{k}, \lambda}\right)\right] \delta(\mathbf{Q} \pm \mathbf{k}-\mathbf{G})
$$

\footnotetext{
14 The $m=0$ term when substituted into equation 5.14 yields the standard time-independent squared-modulus of the crystallographic structure factor.

15 Section 10.3.3 to [2].
} 


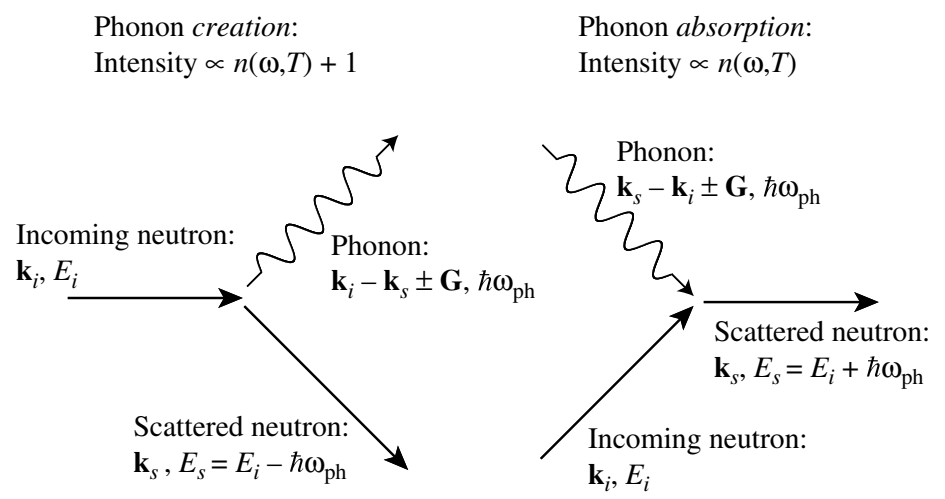

Figure 7. Schematic diagram of the one-phonon neutron scattering process, showing the two cases of creation (left) and absorption (right) of a single phonon, together with the changes in energy and wave vector of the neutron. The vectors $\mathbf{k}_{i}$ and $\mathbf{k}_{s}$ represent the wave vectors of the incident and scattering beams [2].

which is consistent with the detailed balance expressed in equation 5.11, where the structure factor component has the form

$$
F_{\lambda}(\mathbf{Q})=\sum_{j} \frac{b_{j}}{\sqrt{m_{j}}}[\mathbf{Q} \cdot \mathbf{e}(j, \mathbf{k}, \lambda)] \exp \left(i \mathbf{Q} \cdot \mathbf{R}_{j}\right) \exp \left(-W_{j}(\mathbf{Q})\right)
$$

and where $\mathbf{G}$ is any reciprocal lattice vector and $\mathbf{k}$ is the wave vector of the phonon within the Brillouin zone centred on the origin in reciprocal space.

There are a number of points to be made from these equations. First, the scattering is a coupling between the scattering vector and mode eigenvector through the product of the form $\mathbf{Q} \cdot \mathbf{e}$. Moreover, the scattering is also dependent on the structure-factor term, reflecting the details of the crystal structure. These two features mean that measurements with various values of $\mathbf{Q}$ but the same value of $\mathbf{k}$ will have different sensitivities to the different modes with the same $\mathbf{k}$ (that is, different values of $\mathbf{G}$ ). This is of great value in separating different phonon branches from measurements in different places in reciprocal space, making it easier to use predictions to identify best measurements for the different modes in the scattering spectrum. Second, the dependence of the scattering intensity on $\omega^{-1} n(\omega)\left(\propto \omega^{-2}\right.$ for $\left.k_{\mathrm{B}} T>\hbar \omega\right)$ suggests that neutron scattering is most sensitive to lower- $\omega$ modes. Third, the Dirac $\delta$-functions for frequency, $\delta\left(\omega \pm \omega_{\mathbf{k}, \lambda}\right)$, imply that the $m=1$ process involves either absorption (energy gain) or creation (energy loss) of a single phonon of given wave vector. For the absorption process, the probability of scattering is directly proportional to the number of phonons excited. At low temperatures $\left(k_{\mathrm{B}} T<\hbar \omega\right)$, the number of phonons is low and the probability of absorbing a phonon is correspondingly low, but the probability to create a phonon does not vanish at low temperature in the same way (this is the $n+1$ factor). Thus at low temperatures, experiments are usually performed with instrument configuration optimised to measure in energy-gain mode. Overall, the key point from this discussion is that the one-phonon scattering function gives the opportunity to measure individual phonons as a peak in the spectrum when the measurement parameters of $\mathbf{Q}$ and energy change $\hbar \omega$ exactly match those of a phonon, and thereby it is possible to map out the locus of points on a plot of $\omega v s \mathbf{k}$. This is illustrated in Figure 8 for a measurement performed at a constant value of $\mathbf{Q}$ and scanning across $\hbar \omega$; scattering occurs when the locus the the scan crosses a branch in the phonon dispersion curve.

The terms for $m \geq 2$ are not of direct interest for measuring phonon dispersion curves because the conservation laws are sufficient loose that the spectra do not contain clear information that can be easily interpreted. However, the scattering processes corresponding to $m \geq 2$ are seen in experiments and must 


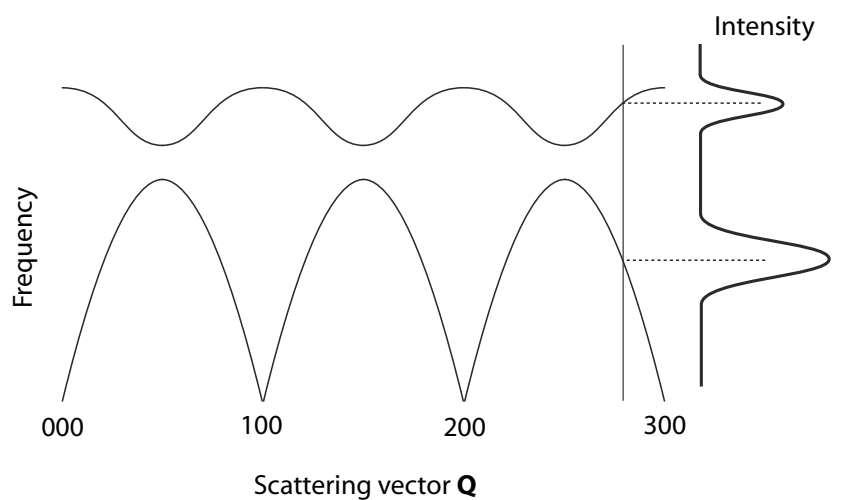

Figure 8. Schematic illustration of the constant- $\mathbf{Q}$ method. The measurement will involve a scan across the energy transfer, which when the $\mathbf{Q}$ and energy transfer values match a phonon there will be a peak in the measured spectrum [2].
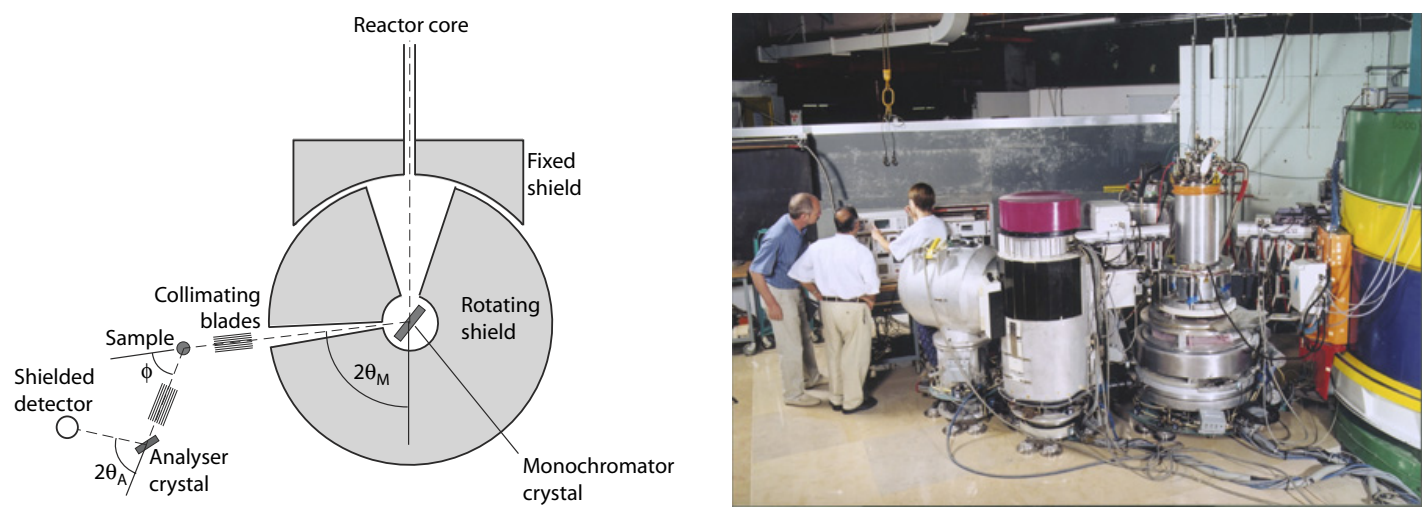

Figure 9. Left, schematic outline of the triple-axis spectrometer [2]. Right, the IN20 triple-axis spectrometer at the Institut Laue Langevin, showing, from left to right, the detector, housing for the analyser crystal, the sample table, and the monochromator drum (photograph courtesy of the Institut Laue Langevin).

be modelled in any attempt to extract quantitative information (eg. fitting mode eigenvectors) from the scattering intensities.

Inelastic scattering of $\mathrm{x}$-ray beams follows exactly the same formalism, except that the scattering lengths $b$ - which are independent of $\mathbf{Q}$ - are replaced by the x-ray form factors $f(|\mathbf{Q}|)$.

\subsection{Experiments to measure dispersion curves: Triple-axis spectrometer}

Having established the basic theory of scattering processes, we now discuss the actual methods used to measure dispersion curves. Traditionally the workhorse instrument has been the triple-axis spectrometer, shown in Figure 9. This was devised at the end of the 1950's for operation on a reactor source of neutrons, which provides intense continuous beams of polychromatic neutrons with velocities of thermal energies. The idea is that the scattering processes involving a single phonon correspond to changes in the wave vector and energy of the neutron beam corresponding to those of the phonon absorbed or created. The triple-axis spectrometer (see the schematic diagram in Figure 9) selects the energy of the incident beam from the continuous spectrum of energies by the angle of scattering from the monochromator crystal (which thereby also defines the incident wave vector). The angle of scattering from the analyser 
crystal defines the energy and modulus of the wave vector of the scattered beam. The difference between the energies of the scattered and incident beam therefore determines the energy of the phonon involved in the scattering process. The wave vector of the phonon also requires the orientation of the scattering to be set, and this is controlled by rotating the angle of scattering from the crystal. Setting these angles requires rotations of the equipment about the axis of the monochromator crystal, which means that the sample and analyser stages need to be able to move easily. In modern spectrometers this is accomplished by the use of air pads with polished flat floors (see the photograph in Figure 9).

One typical mode of operation is the constant- $Q$ method. This is illustrated in Figure 8 . Here the settings are controlled so that the spectrometer measures for a constant value of $\mathbf{Q}$ and scans across energy transfer, observing peaks in the measured signal where the energy corresponds to a phonon $\pm \hbar \omega$. The various angles in the triple-axis spectrometer are not independent, so to perform a constant-Q scan requires two of the three angles to be varied together via computer control [2].

One might remark that there appears to be some redundancy in this equipment, in that three angles are not required to perform a basic scan. In practice, the analyser setting determines the resolution, so is the angle set to a constant value in a constant-Q scan. However, the geometric restrictions on measurements will mean that to scan the full range of energy and wave vector transfers that may be required, it may be necessary to perform constant-Q measurements with different settings of the analyser angle.

\subsection{Experimental results for monatomic crystals}

\subsubsection{General points}

Here we consider crystal structures with only one atom in the primitive unit cell. Examples are many metals crystallising in either the face-centred (e.g. Al, $\mathrm{Pb}$ ) or body-centred (e.g. alkali metals, $\mathrm{Fe}$ ) cubic structures, and also crystals of the rare-gas elements (face-cented cubic). With only three degrees of freedom per unit cell, there will only be three modes per wave vector. These are necessarily the three acoustic modes; there are no optic branches.

\subsubsection{Face-centred cubic elements: Crystalline neon}

The rare-gas solids are useful examples because we know that the forces act only over short distances, and indeed we can model the phonon dispersion curves for all rare-gas solids assuming nearestneighbour interactions only using the simple Lennard-Jones model. Data for crystalline neon [12] are shown in Figure 10.

We highlight a number of points in this figure. First, we note that the plot in Figure 10 actually contains three separate graphs, corresponding to the three sets of wave vectors:

$$
\begin{aligned}
& \mathbf{k}=\xi \mathbf{a}^{*} \equiv(\xi, 0,0) \\
& \mathbf{k}=\xi \mathbf{a}^{*}+\xi \mathbf{b}^{*} \equiv(\xi, \xi, 0) \\
& \mathbf{k}=\xi \mathbf{a}^{*}+\xi \mathbf{b}^{*}+\xi \mathbf{c}^{*} \equiv(\xi, \xi, \xi)
\end{aligned}
$$

where $\xi$ is a number between 0 and $1 / 2$ or 1 , depending on where the Brillouin zone boundary falls. Many phonon dispersion curves are plotted this way. Often the order of the subgraphs reflects a path through reciprocal space. In the reciprocal space of the face-centred cubic lattice, the $\mathbf{k}=(1,0,0)$ Brillouin zone boundary point is simply a lattice translation away from $\mathbf{k}=(1,1,0)$, and thus they are equivalent points. ${ }^{16}$ The graph is drawn in a way that highlights this point, connecting the paths from $\mathbf{k}=(0,0,0)$ to the zone boundary via two directions.

\footnotetext{
16 Recall that due to the face-centred cell being non-primitive, the $h, k, \ell$ points in reciprocal space where $h, k$ and $\ell$ are a mixture of even and odd integers are not true reciprocal lattice vector points.
} 


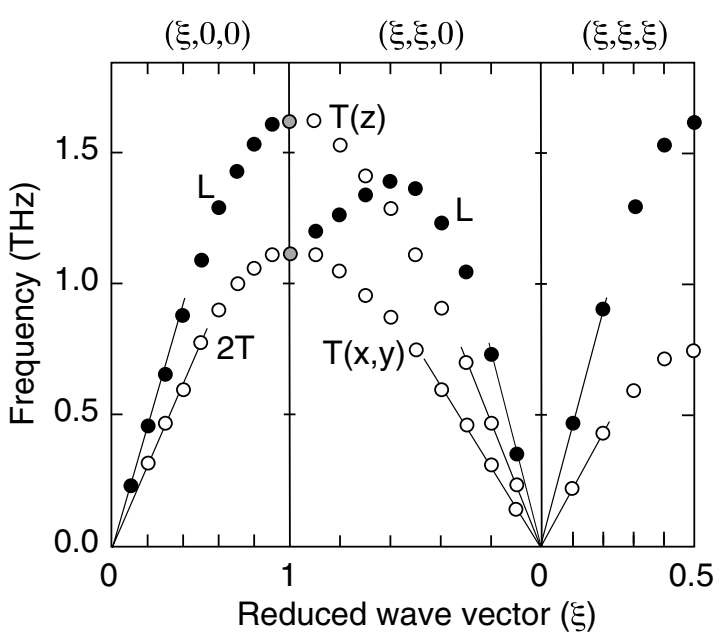

Figure 10. Measurement dispersion curves for neon, face-cented cubic crystal structure, showing data for wave vectors along the three principle symmetry directions. $\mathrm{L}$ and $\mathrm{T}$ denote longitudinal and transverse modes respectively [12].

Next we consider the number of branches in the subgraphs. For $\mathbf{k}=(\xi, \xi, 0)$ we see three sets of data, which correspond to one longitudinal acoustic (LA) mode (atomic displacements in the wave being parallel to $\mathbf{k}$, and two to the transverse acoustic (TA) models (atomic displacements in the two directions orthogonal to $\mathbf{k}$ ). The LA branch is of higher frequency than the TA branch for $\mathbf{k} \rightarrow 0$ along all directions of $\mathbf{k}$; this will be true for all materials, and is a consequence of the elasticity stability conditions.

Finally, we consider the shapes of the curves. For many of the branches, the curves can be seen to closely follow simple sinusoidal curves. This is typically a sign that the Dynamical matrix is dominated by nearest-neighbour interactions. The shape of the dispersion curve for the longitudinal mode along $\mathbf{k}=(\xi, \xi, 0)$ is not a simple sine curve, but has a maximum approximately midway between $\mathbf{k}=0$ and the Brillouin zone boundary. In the case of neon, it can be shown that this maximum arises because there are nearest-neighbour interactions between atoms in both the first and second neighbour planes of atoms normal to $\mathbf{k}=(\xi, \xi, 0)$.

\subsubsection{Body-centred cubic elements: Crystalline potassium}

Figure 11 shows the dispersion curves for potassium for wave vectors along two directions in reciprocal space [13]:

$$
\begin{aligned}
& \mathbf{k}=\xi \mathbf{a}^{*} \equiv(\xi, 0,0) \\
& \mathbf{k}=\xi \mathbf{a}^{*}+\xi \mathbf{b}^{*}+\xi \mathbf{c}^{*} \equiv(\xi, \xi, \xi)
\end{aligned}
$$

As for neon, all bar one of the branches have the appearance of a simple sine curve. For $\mathbf{k}=(\xi, 0,0)$ the longitudinal and transverse curves nearly have the same values; the difference is that there is a second-neighbour interaction causing the longitudinal branch to have slightly higher frequencies midway between $\mathbf{k}=0$ and the Brillouin zone boundary.

In the case of body-centred materials, the Brillouin zone boundaries along the $(\xi, 0,0)$ and $(\xi, \xi, \xi)$ are the points of equivalence. ${ }^{17}$ Unlike for face-cented cubic materials, the longitudinal and transverse modes become degenerate at this point.

\footnotetext{
${ }^{17}$ Recall that due to the body-cented cell being non-primitive, the $h, k, \ell$ points in reciprocal space where $h+k+\ell$ is an odd integer are not true reciprocal lattice vector points.
} 


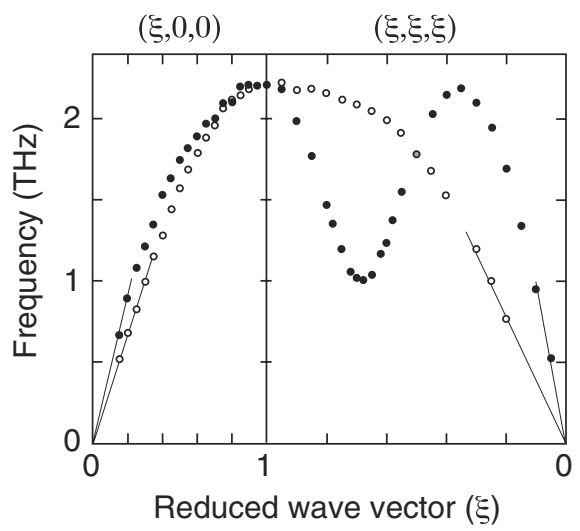

Figure 11. Dispersion curves for potassium, body-centred cubic crystal structure, showing data for wave vectors along two principle symmetry directions [13].

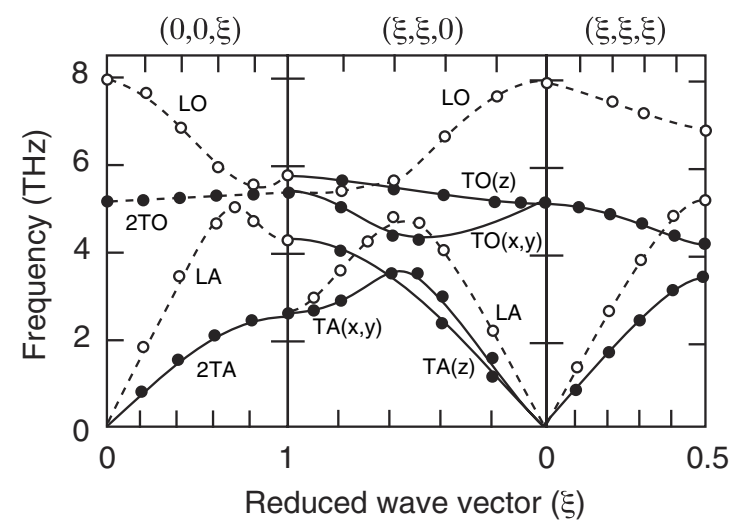

Figure 12. Dispersion curves for $\mathrm{NaCl}$, face-centred cubic crystal structure, showing data for the three principle symmetry directions [14].

The visually interesting feature in figure 11 is the significant dip in the LA branch at around $\mathbf{k}=(2 / 3,2 / 3,2 / 3)$. This arises from the fact that the nearest-neighbour atomic interaction spans across to the atoms in the third-neighbour plane of atoms, and this is strongest than the interactions involving atoms in the first and second-neighbour planes because the force acts directly along the $\mathrm{K}-\mathrm{K}$ bond. It is because this is the third-neighbour plane that the dip occurs at the point it does. At this wave vector, the atoms along a chain parallel to $(\xi, \xi, \xi)$ all move together without any change in the $\mathrm{K}-\mathrm{K}$ distance.

\subsection{Experimental results for simple ionic solids}

We now consider a material with two atoms in the unit cell, which is the point where we started the theory. Specifically we consider $\mathrm{NaCl}$, and the dispersion curves are shown in Figure 12 [14].

The key new feature over the monatomic solids, and anticipated by the theory described in Section 2.4 , is the presence of the three optic modes for each wave vector, although in Figure 12 the transverse optic modes along the [001] and [111] directions are degenerate in the same way that there are degeneracies for the transverse acoustic modes. 

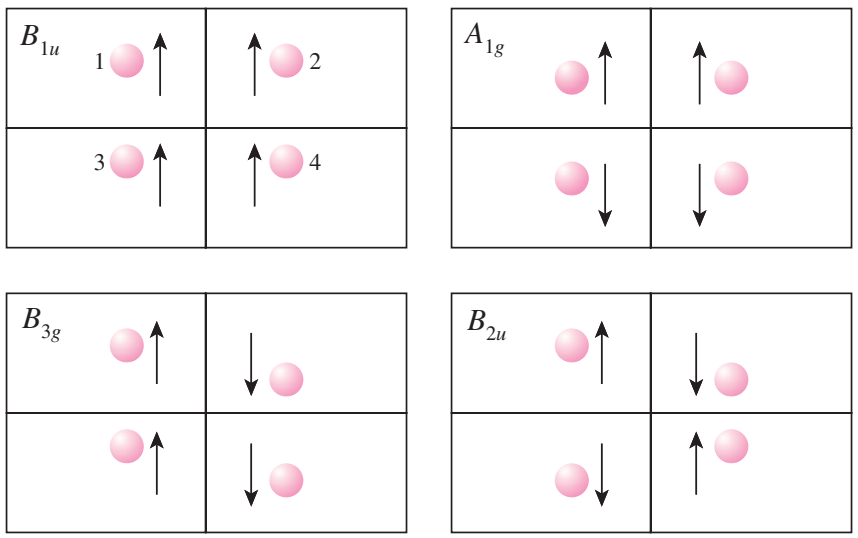

Figure 13. Examples of four modes of different symmetries for a model with one atom in the unit cell of symmetry $m m m[2]$.

Let us first consider the [111] direction. Here the dispersion curves for the pairs of transverse and longitudinal modes look very similar to the dispersion curves for the one-dimensional model shown in Figures 2 and 4. The crystal structure of the $\mathrm{NaCl}$ structure shows alternative layers of $\mathrm{Na}$ cations and $\mathrm{Cl}$ anions normal to the [111] direction, so the similarities with the one-dimensional model with nearest-neighbour interactions is not surprising.

Now we consider the [001] direction. Since each layer normal to [001] consists of both Na cations and $\mathrm{Cl}$ anions we might not expect any similarities between the experimental dispersion curves and those of the simple model considered earlier. Before we think about the detailed shapes of the dispersion curves, we should consider one simple fact that we glossed over in the discussion about the [111] direction. Consider the special case for $\mathbf{k}=0$, but think about the fact that in the limiting case $\mathbf{k} \rightarrow 0$ we can define a direction for $\mathbf{k}$ and thus also define transverse and longitudinal waves. In the exact limit of $\mathbf{k}=0$, but still thinking about the direction of $\mathbf{k}$, a little thought will suggest that the atomic motions associated with the longitudinal optic mode for $\mathbf{k} \|$ [001] will be equivalent to those generated by one of the transverse optic modes for $\mathbf{k} \perp$ [001]. Thus one might expect the frequencies of the longitudinal and transverse optic modes to be the same at $\mathbf{k}=0$, and indeed some lattice dynamics codes will predict this for the exact point $\mathbf{k}=0$. But this is a case where we need to think about limiting values rather than exact points; that is, we need to think about $\mathbf{k} \rightarrow 0$ rather than $\mathbf{k}=0$. And here we immediately see a difference between the TO and LO modes, namely that the polarisation fields generated by the two modes are perpendicular and parallel to the direction of $\mathbf{k}$ respectively. This is sufficient to generate differences in the dynamical matrix as $\mathbf{k} \rightarrow 0$, but which are not captured in a simple implementation of the dynamical equations exactly at $\mathbf{k}=0$. Modern lattice dynamics codes now implement an appropriate correction, but the reader is warned to be very careful to check this feature with their preferred code. ${ }^{18}$

A second point to note from the dispersion curves along [001] is the way in which the LA and LO modes appear to want to cross at $\xi \sim 0.7$, but instead of crossing the two curves get close but then 'repel' each other. This feature is known as anti-crossing, and occurs when two modes of the same symmetry appear to be trying to cross.

Which brings us to the issue of symmetry. The atomic motions associated with any normal mode will break some of the symmetry operations and preserve other symmetry operations. Consider the simple example of one-dimensional motions in a crystal of symmetry $\mathrm{mmm}$, Figure 13 . The mean

18 The author's experience is that researchers often fail to heed this warning. He has examined one PhD thesis where this effect was not taken into account, meaning that many of the results discussed were simply wrong. 

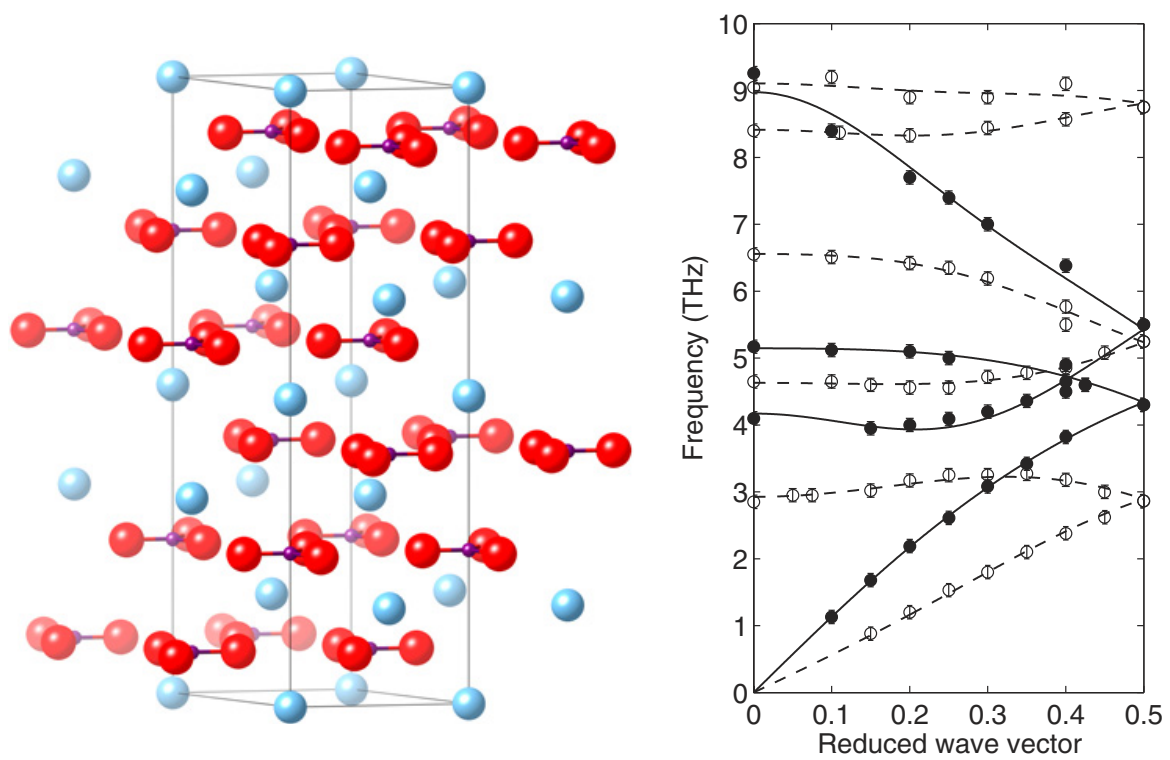

Figure 14. Crystal structure (left) and phonon dispersion curves (right) for calcite, $\mathrm{CaCO}_{3}$, for wave vectors along the crystal three-fold axis. This plot shows the low-frequency spectrum in which the carbonate groups move as rigid molecular units [15].

positions of the atoms are consistent with the $\mathrm{mmm}$ symmetry. The four permutations of atomic motion, which are labelled by their effects on symmetry - formally called the irreducible representaions of the symmetry group - are $B_{1 u}=[\uparrow, \uparrow, \uparrow, \uparrow], A_{1 g}=[\uparrow, \uparrow, \downarrow, \downarrow], B_{3 g}=[\uparrow, \downarrow, \uparrow, \downarrow]$ and $B_{2 u}=[\uparrow, \downarrow, \downarrow, \uparrow]$. These preserve or break some of the symmetry operations, as can be seen from the relevant subset of the $\mathrm{mm}$ character table (that is, the subset that contains the symmetry operations that act within the plane of the diagram):

\begin{tabular}{c|ccccc} 
& 1 & $m_{1}$ & $m_{2}$ & $\overline{1}$ & 2 \\
\hline$A_{1 g}$ & +1 & +1 & +1 & +1 & +1 \\
$B_{3 g}$ & +1 & -1 & -1 & +1 & +1 \\
$B_{1 u}$ & +1 & +1 & -1 & -1 & -1 \\
$B_{2 u}$ & +1 & -1 & +1 & -1 & -1
\end{tabular}

The $B_{1 u}$ mode is the acoustic mode (all atoms move in phase), and it can be seen that it breaks the centre of symmetry in addition to the mirror symmetry whose plane normal is parallel to the displacement direction. The $A_{1 g}$ mode preserves the complete symmetry, and corresponds to an optic mode. The $B_{3 g}$ optic mode breaks both of the mirror symmetries, but preserves the centre of symmetry and the 2-fold rotation axis normal to the plane. The $B_{2 u}$ optic mode has similar effect on symmetry as the $B_{1 u}$ acoustic mode, except that the mirror symmetry broken is that where the plane normal is perpendicular to the displacement direction.

Thus in a crystal containing several atoms in the unit cell, the various modes (optic and acoustic) can be labelled according to their effect on the symmetry - noting that the important symmetry is not the symmetry of the crystal per se but the symmetry as lowered by the imposition of the wave vector on the crystal, so that in the case of $\mathbf{k} \|[100]$ in $\mathrm{NaCl}$ the symmetry will be $m m 4$ rather than the full $m \overline{3} m$ group of the crystal.

Finally we consider $\mathbf{k} \|$ [110]. Here there are no degeneracies, and we also see the anti-crossing effects discussed above as the frequencies of the acoustic and optic modes come close in value. 

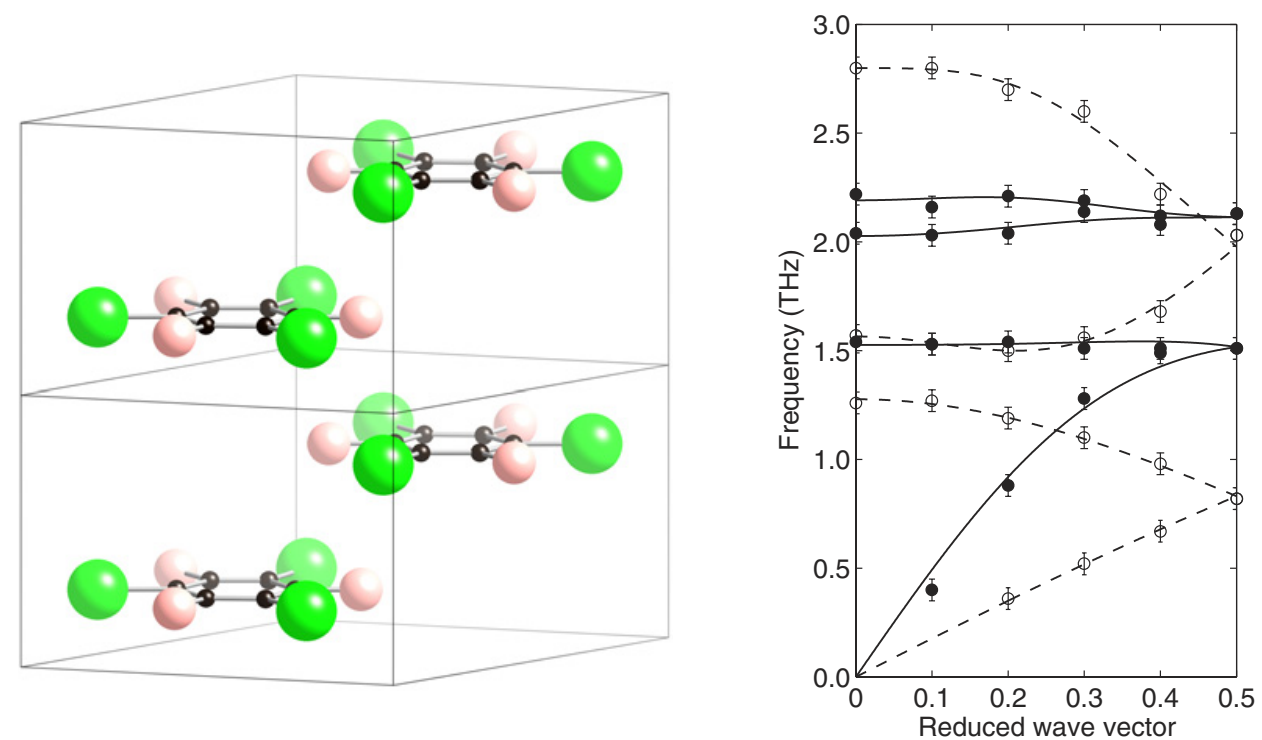

Figure 15. Crystal structure [16] (left) and phonon dispersion curves (right) for the molecular crystal $\mathrm{C}_{6} \mathrm{~F}_{3} \mathrm{Cl}_{3}$. This plot shows the low-frequency spectrum in which the molecules move as rigid objects [17].

\subsection{More complex crystals}

The generalisation from $\mathrm{NaCl}$ with two atoms in the primitive unit cell to the more general case is straightforward. With $z$ atoms in the unit cell, there will be 3 acoustic modes and $3 z-3$ optic modes for any wave vector.

In Figure 14 we show measurements of the dispersion curves for calcite, $\mathrm{CaCO}_{3}$, measured along the 3-fold axis of the rhombohedral space group [15] (the [111] direction in the rhombohedral setting of the unit cell, or the [001] direction in the hexagonal setting). Calcite has 2 formula units in the unit cell, hence $z=10$. But of the 30 modes, 12 will involve deformations of the rigid $\mathrm{CO}_{3}^{2-}$ carbonate groups and have high frequencies. The remaining 18 lower-frequency modes will involve translations of the $\mathrm{Ca}$ cations and translations and rotations of the carbonate groups. These are the modes shown in Figure 14, although the transverse modes are degenerate so that only 12 branches are shown.

A similar situation exists in molecular crystals. In Figure 15 we show the dispersion curves for the molecular crystal $\mathrm{C}_{6} \mathrm{~F}_{3} \mathrm{Cl}_{3}$ [17], together with the crystal structure (hexagonal symmetry). The crystal has 2 molecules in the unit cell, and thus has 12 modes that involve rigid-body motions of the molecules and 60 higher-frequency modes involving deformations of the molecules. The dispersion curves for the low-frequency rigid-molecule modes are what are shown in Figure 15. Again, for wave vectors parallel to the 3-fold axis (the [001] axis) there are degeneracies of the transverse modes.

\section{CALCULATIONS OF DISPERSION CURVES}

\subsection{Model simulations}

In the early years of measuring dispersion curves by neutron scattering, the values of the parameters in the dynamical matrix, namely the force constants $\phi_{0, h^{\prime}}^{j, j^{\prime}}$, were adjusted by a least-squares method to give the best agreement between calculated frequencies and experiment. It was found that for many ionic crystals best results were obtained by taking account of ionic polarisability. One simple approach to achieve this is through the use of the shell model, as illustrated in Figure 16. Essentially the ions 


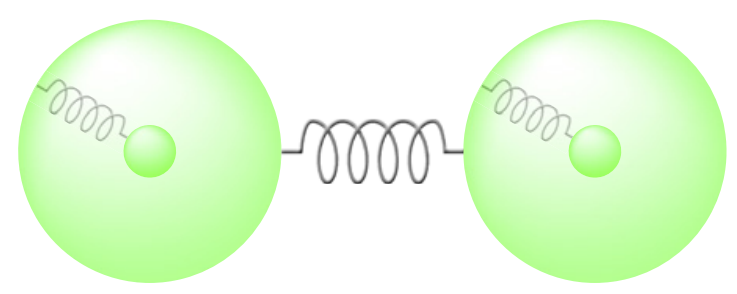

Figure 16. Representation of the shell model, showing the small core and spherical shells components. The spring connecting the core and shell represents the interaction between these two components. Usually the short-range interactions (for example, the Buckingham interaction of equation 6.1) between neighbouring atoms are assumed to involve only the shells, and this is represented schematically by the large spring connecting two shells [2].

are represented by two components, namely the core representing the nucleus and inner electrons, and the shell representing the outer electrons. Both core and shell are charged, and typically interact via a simple harmonic potential. ${ }^{19}$ The shell is treated as having no mass, so the dynamical equations are written in a way that assumes that the positions of the shells relax instantaneously when the positions of interacting neighbouring atoms change (the adiabatic approximation). Because the shells represent the outer electrons, it is often assumed that the interatomic interactions are represented by interactions between neighbouring shells. More sophisticated versions of the shell model have been developed.

Over the past few years it has become more common to compute the force constants from models of the interatomic interactions based on parameterised functions. The simplest models for ionic crystals include Coulomb interactions, shell models, and short-range potential energy functions of the form

$$
\text { - } E(r)=A \exp (-r / \rho)-C r^{-6}
$$

where $A, \rho$ and $C$ and parameters, whose values may be determined by several approaches. This is called the Buckingham potential. The $-r^{-6}$ term is the attractive interaction which physically arises from correlated dipolar fluctuations of the electrons of the interacting atoms, and is known as the Dispersion, van der Waals or London interaction. ${ }^{20}$ The coefficient $C$ can in fact be calculated from quantum chemistry methods, being related to the atomic polarisability. The exponential term - called the Born-Mayer interaction - is the repulsive part of the function, and arises from overlap of the electron density of the two atoms. The repulsive interaction can be calculated by quantum mechanics methods, and although we can't derive this function from first principles it turns out to give a reasonably good description of such calculations. Another model is the Morse potential, which has the form

$$
E(r)=E_{0}\left(\exp \left(2 \alpha\left(r_{0}-r\right)\right)-2 \exp \left(\alpha\left(r_{0}-r\right)\right)\right)
$$

By design, this potential is good for modelling covalent bonds, and can encompass bond energy $\left(E_{0}\right)$, bond length $\left(r_{0}\right)$ and curvature of the potential energy $(\alpha)$ directly. However, the parameters lose their direct connection with these physical concepts when used in conjunction with the Coulomb interaction between the two atoms - whilst it might seem preferable to set the Coulomb interaction to zero for this specific bond, it is turning out from experience that including the Coulomb interaction can give better overall results in modelling materials with mixed ionic and covalent bonds. We will see an example below (Section 6.2).

One way to deduce parameter values, including values of the ionic charges and the spring constants in the shell model, is to adjust them from a starting model to improve the model predictions of crystal

\footnotetext{
19 More sophisticated models are available, such as models with higher-order terms for the core-shell interaction in the interaction potential, with anisotropic potentials, or with variable size and shape.

20 Sometimes the $-r^{-6}$ term is included with a term of the form $+r^{-12}$ in a function called the Lennard-Jones potential. Whilst this function has a pleasant appearance, and can be nice to work with for certain test and pedagogical purposes, this author doesn't particularly like it for research applications. There are nevertheless simulation communities who do use this function regularly.
} 


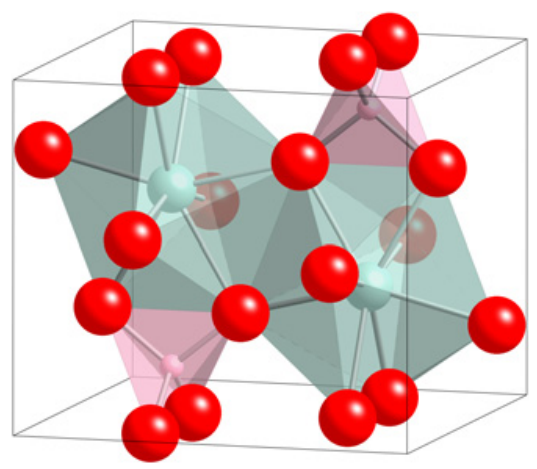

Figure 17. Crystal structure of $\mathrm{YPO}_{4}$ [19], highlighting the $\mathrm{PO}_{4}$ tetrahedra and the irregular $\mathrm{YO}_{8}$ polyhedra. The tetragonal $z$ axis is along the vertical direction.

structure and properties - including vibrational frequencies - with experimental data. More recently it has become possible to adjust these parameters to give best agreement with quantum mechanical calculations, for example by fitting to energies of multiple configurations.

An example of a modern simulation code for the computation of lattice properties including crystal structure, physical properties (dielectric, piezoelectric and elastic constants) and dispersion curves is GULP [18]. This program enables the user to prepare an input file containing details of the crystal structure and the parameters for the model interatomic potentials with minimum of effort. Simple directives in the input file can be used to instruct GULP to adjust the crystal structure to give the lowest lattice energy, ${ }^{21}$ to compute values of physical properties, and to calculate vibrational frequencies at any given wave vector or for a specified range of wave vectors, and finally to compute thermodynamic properties based on the vibrational frequencies (see below).

\subsection{Example of calculations with an empirical model: $\mathrm{YPO}_{4}$}

The mineral xenotime, $\mathrm{YPO}_{4}$, crystallises with a tetragonal unit cell, Figure 17 [19]. We have developed an interatomic model potential for xenotime designed to obtain the best prediction of crystal structure and elastic constants. Our approach was to use a rigid ion model, with a Morse potential, equation (6.2), to describe the $\mathrm{P}-\mathrm{O}$ interaction, a Born-potential, (the exponential term in equation (6.1)) to describe the $\mathrm{Y}-\mathrm{O}$ interaction, and a Buckingham potential equation 6.1 to describe $\mathrm{O}-\mathrm{O}$ interactions, with Coulomb interactions between all atoms (including neighbouring pairs of atoms modelled by the Morse potential). GULP was used to fit the model parameters to give best agreement between calculated and experimental crystal structure and elastic constants. The best model has the following parameters:

\begin{tabular}{c|ccccccc} 
& \multicolumn{3}{|c}{ Morse potential $($ eq 6.2) } & \multicolumn{3}{c}{ Bunkingham potential (eq 6.1) } & Coulomb \\
& $D(\mathrm{eV})$ & $r_{0}(\AA)$ & $\alpha\left(\AA^{-1}\right)$ & $A(\mathrm{eV})$ & $\rho(\AA)$ & $C\left(\mathrm{eV} . \AA^{6}\right)$ & $Q_{1}(|e|)$ \\
\hline $\mathrm{P}-\mathrm{O}$ & 70.86 & 2.83 & 1.555 & & & & 1.2556 \\
$\mathrm{Y}-\mathrm{O}$ & & & & 15949 & 0.2114 & 0.0 & 2.1272 \\
$\mathrm{O}-\mathrm{O}$ & & & & 770.8 & 0.3436 & 55.0 & -0.8457
\end{tabular}

\footnotetext{
21 The lattice energy is defined as the potential energy of the crystal with all atoms at rest, which corresponds to the energy of the classical crystal at a temperature of $0 \mathrm{~K}$.
} 


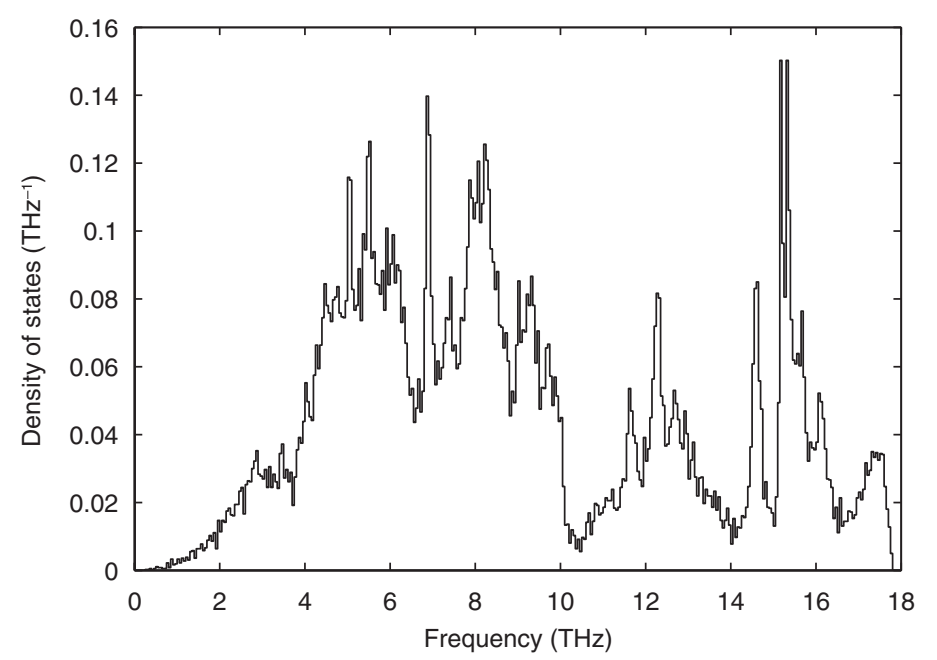

Figure 18. Density of states for $\mathrm{YPO}_{4}$.

This model gave the following results, comparing calculated and experimental values for some structural details (lattice parameters and bond lengths) and elastic constants $c_{i k}$ :

\begin{tabular}{|c|c|c|c|c|c|c|c|}
\hline & \multicolumn{2}{|c|}{ Lattice parameters } & \multicolumn{3}{|c|}{ Cation-oxygen bond lengths } & & \multirow[b]{3}{*}{$\AA$} \\
\hline & $a$ & $c$ & $\mathrm{P}-\mathrm{O}$ & $\mathrm{Y}-\mathrm{O}(1)$ & $\mathrm{Y}-\mathrm{O}(2)$ & & \\
\hline Expt & 6.8947 & 6.0276 & 1.540 & 2.309 & 2.381 & & \\
\hline \multirow[t]{3}{*}{ Calc. } & 6.8957 & 6.0280 & 1.554 & 2.309 & 2.350 & & $\AA$ \\
\hline & \multicolumn{6}{|c|}{ Intrapolyhedral $O-O$ distances } & \\
\hline & $\mathrm{O}-\mathrm{O}(\mathrm{P} 1)$ & $\mathrm{O}-\mathrm{O}(\mathrm{P} 2)$ & $\mathrm{O}-\mathrm{O}(\mathrm{Y} 1)$ & $\mathrm{O}-\mathrm{O}(\mathrm{Y} 2)$ & $\mathrm{O}-\mathrm{O}(\mathrm{Y} 3)$ & & \\
\hline Expt. & 2.409 & 2.566 & 2.958 & 2.801 & 3.355 & & $\AA$ \\
\hline \multirow[t]{3}{*}{ Calc. } & 2.394 & 2.607 & 2.961 & 2.748 & 3.346 & & $\AA$ \\
\hline & \multicolumn{6}{|c|}{ Elastic constants } & \\
\hline & $c_{11}$ & $c_{33}$ & $c_{12}$ & $c_{13}$ & $c_{44}$ & $c_{66}$ & \\
\hline Expt. & 220 & 332 & 55 & 86 & 65 & 17 & GPa \\
\hline Calc. & 225 & 328 & 35 & 97 & 62 & 17 & $\mathrm{GPa}$ \\
\hline
\end{tabular}

It is worth remarking that the level of agreement between calculated and experimental values is possibly better than we have a right to expect, but interestingly the agreement was better than with a model based only on Buckingham potentials, even though Buckingham potentials have proven to be highly successful in modelling silicates. There are two interesting things about this structure from the modelling perspective. First is that the yttrium has a irregular coordination, and some models have problems with irregularities in crystal structures. Second, the $c_{66}$ elastic constant can be very hard to calculate with good agreement with experiment, as was found for modelling the related mineral zircon, $\mathrm{ZrSiO}_{4}[20]$.

The phonon density of states calculated using this model is shown in Figure 18 up to the upper frequency of the continuous range; there are higher frequencies involving deformations of the structural polyhedra. This figure shows several important characteristic features of the density of states. The first is the variation with $\omega^{2}$ at low frequency, characteristic of the acoustic modes as deduced in the original Debye model, equation 4.6. The second feature is the various peaks associated with the bands of optic modes, some of which are quite tight, implying that the dispersion curves for some branches have little dependence on wave vector. 


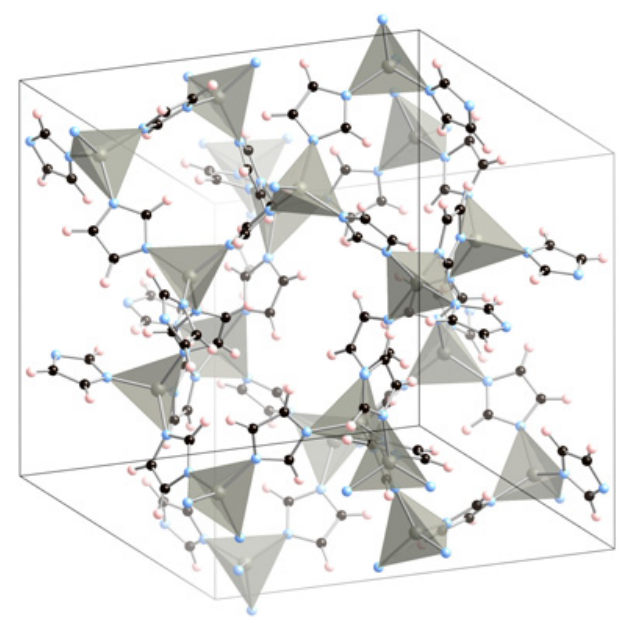

Figure 19. Crystal structure of one of the phases of zinc imidazolate, $\mathrm{Zn}\left[\mathrm{C}_{3} \mathrm{~N}_{2} \mathrm{H}_{3}\right]_{2}$, ZIF-4, highlighting the network of $\mathrm{ZnN}_{4}$ tetrahedra linked via the imidazolate ligands in an open frameworks structure [21].

\subsection{Empirical model for zinc imidazolate, $\mathrm{Zn}\left[\mathrm{C}_{3} \mathrm{~N}_{2} \mathrm{H}_{3}\right]_{2}$}

There is a rapidly growing interest in a group of materials known as metal-organic framework (MOF) structures. There are characterised as containing metal cations that are connected together into a periodic framework through bridging organic ligands, in exactly the same way that silicon atoms are linked together through bridging oxygen atoms in the phases of silica, $\mathrm{SiO}_{2}$. One family of MOF structures consist of metals such as zinc bridge by the imidazolate, $\left[\mathrm{C}_{3} \mathrm{~N}_{2} \mathrm{H}_{3}\right]^{-}$, ligand to form structures that are analogous to zeolitic silica structures; an example is shown in Figure 19.

The problem in modelling such structures is that apart from the crystal structure there is a shortage of experimental data against which to tune the interatomic potentials, compounded by the fact that there are many interatomic interactions to be considered. Our approach was to use components of transferable models where possible - a transferable model is one that can be applied robustly to many members of the same family of materials, such as organic and silicate crystals - and to use quantum chemical approaches to compute values for parameters for interactions specific to this material. The intramolecular interactions (for example, those describing the $\mathrm{C}-\mathrm{N}, \mathrm{C}-\mathrm{H}$ and $\mathrm{C}-\mathrm{C}$ bonds within the imidazolate ligand, together with additional bond-bending and torsional interactions to define the shape of the molecule) were taken from the MM3 molecular mechanics model [22], and the intermolecular interactions were taken from the transferable models developed by Williams [23] for non-bonded interactions in crystals of organic molecules. The $\mathrm{Zn}-\mathrm{N}$ interactions and the orientational $\mathrm{Zn}-\left[\mathrm{C}_{3} \mathrm{~N}_{2} \mathrm{H}_{3}\right]^{-}$interactions were computed from quantum chemistry calculations using the Dalton code [24], and the distribution of atomic charges was derived from a distributed multipole analysis based on computed electronic wave functions [25]. The resultant model was therefore derived without fitting to experimental data, which will mean that it is not reasonable to expect it to give the same accuracy as obtained for $\mathrm{YPO}_{4}$ above. Moreover, the open framework structure has a high degree of flexibility, meaning that the large differences between experimental and calculated crystal structure are possible with little change in energy. Indeed, convergence to the lowest energy structure in a lattice energy minimisation calculation for this model takes an inordinately large number of steps (of order a few thousands, compared to the usual order of a few tens).

Figure 20 shows the phonon density of states computed for zinc imidazolate. This material has much smaller elastic constants than $\mathrm{YPO}_{4}$ (computed values $c_{11}=8.0, c_{22}=6.2, c_{33}=7.0, c_{44}=1.9$, $c_{55}=2.4, c_{66}=3.3 \mathrm{GPa}$, other terms zero or small in value), so that the $\omega^{2}$ component of the density 


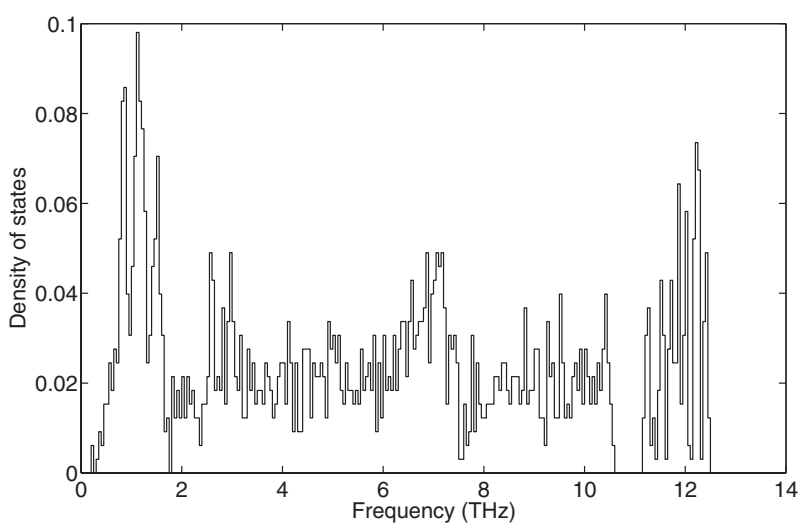

Figure 20. Density of states for zinc imidazolate ZIF-4 structure using the potential model described in the text.

of states is much shallower than for $\mathrm{YPO}_{4}$. The important low-frequency vibrations that contribute to the density of states are instead the modes that flex the structure without the molecule or $\mathrm{ZnN}_{4}$ units deforming.

\subsection{Quantum mechanical calculations}

One of the great achievements in modern condensed matter sciences, aided to a large extent by the considerable growth of available computing, has been the development of methods to compute the electron density and properties of matter using quantum mechanics. The most common approach is called Density Functional Theory (DFT), named after the fact that the core equations are written in terms of the electron density rather than the electronic wave functions. For practical purposes, it is often assumed that the electrons close to atomic nuclear are tightly bound and are capable of being represented by a potential energy function - a pseudopotential - rather than being required to be treated explicitly. The current state of the art has been well described in several recent text books [26].

Early calculations with quantum mechanical approaches used the frozen phonon approximation, where atoms were displaced in accord with a chosen eigenvector and the plot of energy as a function of displacement amplitude converted into a value of frequency. This approach works if the eigenvectors are wholly determined by symmetry, as might be the case in simple ionic crystals, but for many materials the eigenvectors are not known in advance.

A more sophisticated approach that followed was to compute the force constants between individual atoms by displacing pairs of atoms and calculating the change in energy. For pairs of atoms not in the same unit cell, this was accomplished by expanding the size of the simulation sample in the corresponding directions to create a supercell [27].

The frozen phonon and supercell approaches require numerical computations of the energy surface that contributes to the dynamical matrix, and will only give rise to a specific set of wave vectors. Modern approaches now use a method called linear response, where the values of the force constants can be computed analytically from the wave functions via a perturbation theory approach, and allow computations for any desired wave vector.

\subsection{Example of calculation using DFT with the linear response approach: $\mathrm{Cu}_{2} \mathrm{O}$}

$\mathrm{Cu}_{2} \mathrm{O}$ has a relatively simple crystal structure, shown in Figure 21. This material is interesting for a number of potential applications, but our interest has focussed on the negative thermal expansion. 

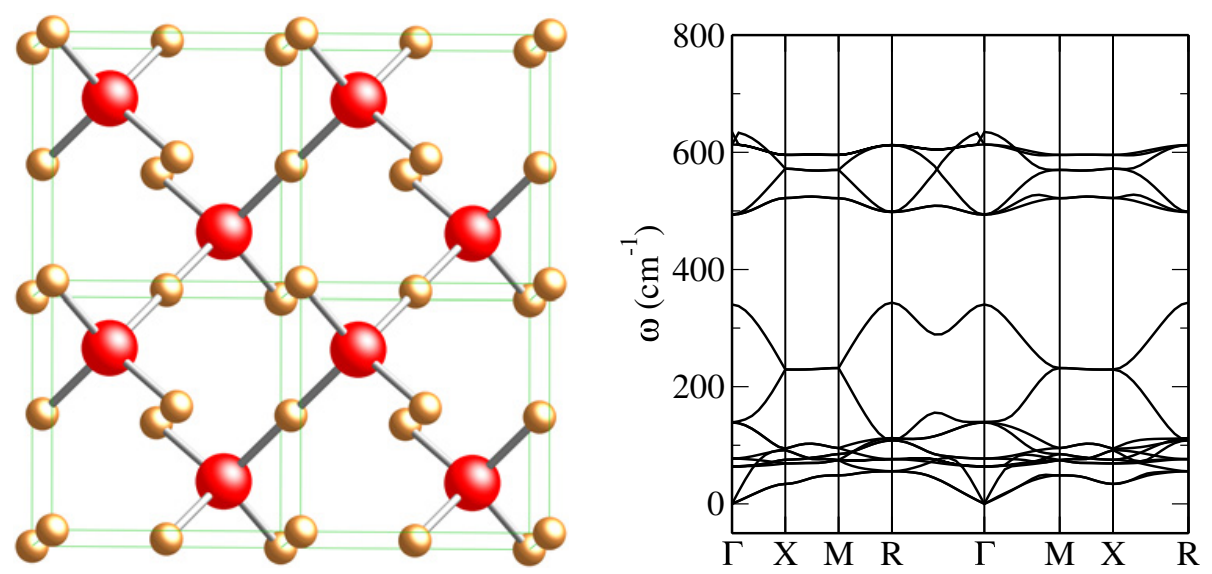

Figure 21. Left, crystal structure of $\mathrm{Cu}_{2} \mathrm{O}$, showing $\mathrm{OCu}_{4}$ tetrahedra linked via corners to form two interpenetrating three-dimensional networks. Right, phonon dispersion curves of $\mathrm{Cu}_{2} \mathrm{O}$ calculated by Density Functional Theory methods using linear response. Here specific points in reciprical space are labelled using conventional letters: $\Gamma=(0,0,0), \mathrm{X}=(1 / 2,0,0), \mathrm{M}=(1 / 2,1 / 2,0)$ and $\mathrm{R}=(1 / 2,1 / 2,1 / 2)$.

Thermal expansion is typically described by the equation derived by minimisation of the sum of crystal lattice energy and phonon free energy Section 11.3 of [2]:

$$
\beta=\frac{1}{V}\left(\frac{\partial V}{\partial T}\right)_{P}=\frac{K_{T} \gamma C_{V}}{V}
$$

where $V$ is the volume, $C_{V}$ is the constant-volume heat capacity, and $K_{T}$ is the isothermal compressibility defined as

$$
K_{T}=-\frac{1}{V}\left(\frac{\partial V}{\partial P}\right)_{T}
$$

$\gamma$ is the quantity known as the Grüneisen parameter, and is defined as

$$
\gamma=\frac{1}{C_{V}} \sum_{i} \gamma_{i} \hbar \omega_{i} \frac{\partial n\left(\omega_{i}, T\right)}{\partial T}
$$

where the individual quantities known as the mode Grüneisen parameters are defined as

$$
\gamma_{i}=-\frac{V}{\omega_{i}} \frac{\partial \omega_{i}}{\partial V}=-\frac{\partial \ln \omega_{i}}{\partial \ln V}
$$

The mode Grüneisen parameters are the key quantities in this theory. In physical terms, a lowering of the mode frequencies with volume will lower the entropy and hence favour the volume change at high temperature, and vice versa. In most cases $\gamma_{i}$ has a value of around +1 . For negative thermal expansion, there is a need for sufficient number of modes to have negative values of $\gamma_{i}$.

In the harmonic approximation, all values of $\gamma_{i}$ are exactly zero, because the values of the interatomic force constants $\left(\phi_{n, n^{\prime}}^{j, j^{\prime}}\right.$ in equation (2.35)) are independent of interatomic distance. We understand the origin of positive values of $\gamma_{i}$ in most cases by consideration of the typical form of the interatomic potential for nearest-neighbour interactions illustrated in Figure 1. The asymmetry of the function in the vicinity of the minimum means that the force constants become softer on expansion of the bond and stiffer on compression, and hence lower vibrational frequencies for larger volumes. Negative values of the mode Grüneisen parameters can instead arise from geometric effects [28, 29], particularly when the existence of strong bonds define identifiable polyhedral groups of atoms that can move as if they were 


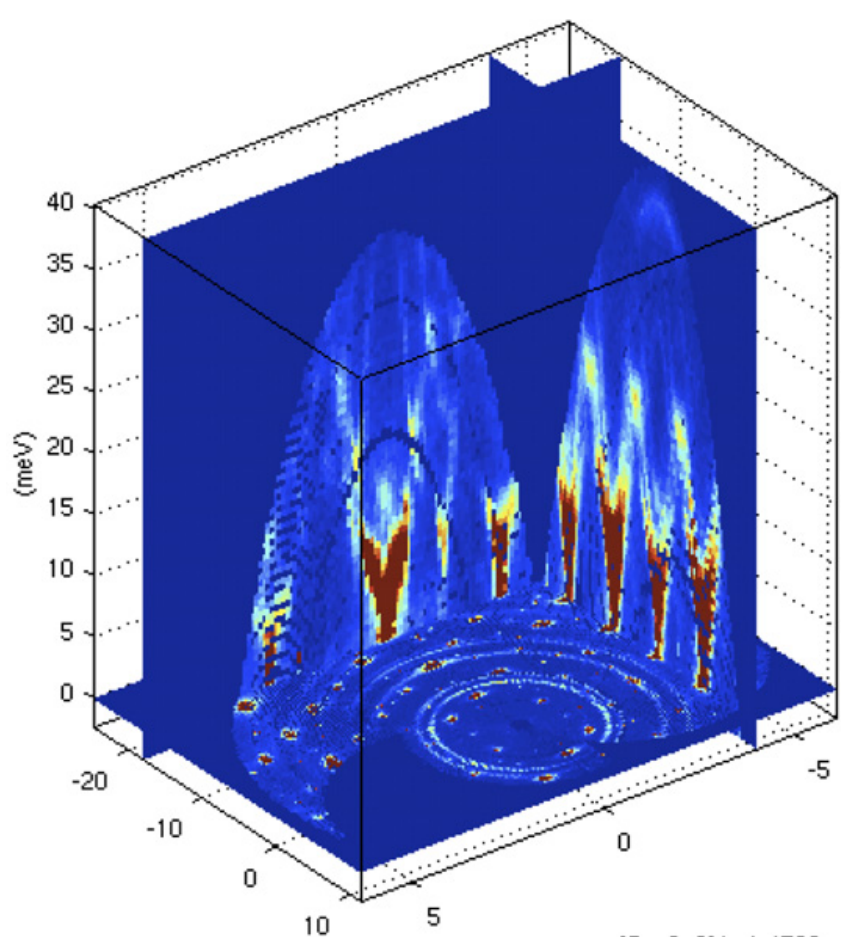

Figure 22. View of the three-dimensional measurement of inelastic neutron scattering from calcite using the MERLIN spectrometer at ISIS. The base plane is for zero energy transfer, showing only the Bragg peaks. The two vertical cuts correspond to the phonon wave vector lying in the $\mathbf{a}^{\star}$ and $\mathbf{c}^{\star}$ planes. The dominant modes that can be seen are the acoustic modes and some optic branches.

rigid units. The important motions for negative thermal expansion are rotations of these group, which may have low frequency if the linkages between polyhedra are rather less stiff. Rotational motions have the effect of pulling the structure inwards, an effect that increases with higher temperature due to the increase in amplitude of the rotational vibrations. This has been documented in detail elsewhere [29], and such effects are often the origin of negative thermal expansion in materials.

In the case of $\mathrm{Cu}_{2} \mathrm{O}$, DFT calculation of the phonon dispersion curves using linear response have been performed by Bohnen and colleagues [30] and ourselves (unpublished). Our results are shown in Figure 21. The important modes for negative thermal expansion are the lowest-frequency optic mode, which are represented by a triply-degenerate vibration at $\mathbf{k}=0$ (labelled $\Gamma$ in Figure 21), and one of the acoustic modes. Both modes have a significant negative value of its Grüneisen parameter, with the optic branch being confirmed by spectroscopic measurements. We have found that the origin of this for the acoustic mode is a pressure-induced instability, which would lead to a ferroelastic phase transition. We have found that the optic mode with the negative Grüneisen parameter has a significant contribution to the atomic displacements from whole-body rotations of the $\mathrm{CuO}_{4}$ tetrahedra.

\section{COMBINING EXPERIMENTS AND CALCULATIONS}

Modern spectrometers for measurements of phonons for both inelastic neutron and $\mathrm{x}$-ray scattering now permit construction of maps of phonon scattering intensity across the space spanned by $\mathbf{Q}$ and energy transfer. Indeed, the MERLIN spectrometer at ISIS [31] automatically gives data for three-dimensional volumes of $\mathbf{Q}$. An example is shown in Figure 22. 

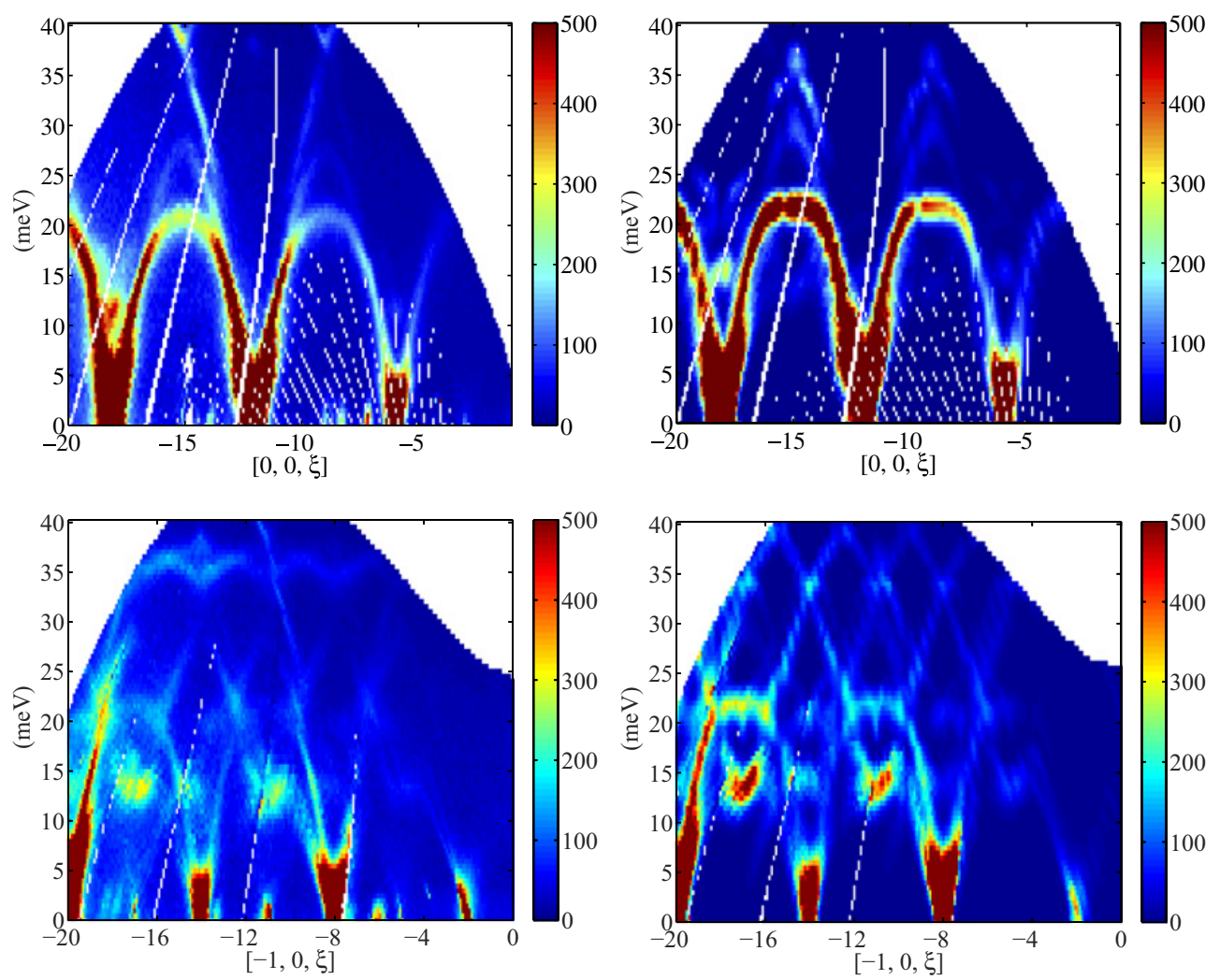

Figure 23. Two sections of the $\mathbf{Q}-E$ scattering surface for calcite with wave vectors along the $\mathbf{c}^{s}$ tar direction measured on the MERLIN spectrometer at ISIS, left, and calculated using an empirical model [33], right.

These multi-dimensional maps are sufficiently rich in detail that increasingly we need matching calculations to enable interpretation. We have developed methods to compute the neutron scattering maps taking account of resolution and course corrections for multiphonon and multiple scattering; similar methods have also been developed for inelastic x-ray scattering [32]. These methods are able to interpret experiments performed using single crystals, and also using polycrystalline samples. Our hope had been that with the polycrystalline case it would be possible to refine a force-constant model, but indications are that there is not a unique solution for materials more complex than monatomic solids. On the other hand, fitting to the single-crystal data should be possible, but at this point in time it appears to be computationally too demanding a task.

Examples of experimental data and matching simulations are shown in Figure 23, where we compare measurements of the phonon dispersion curves in calcite obtained from the MERLIN spectrometer at ISIS with our calculations using an empirical model [33]. It can be seen that the agreement between measurement and calculation is good. This comparison has enabled us to deduce the phonon dispersion curves for wave vectors along $\mathbf{a}^{\star}$ from the data set (not shown here).

\section{Acknowledgements}

I am grateful to the following collaborators: Beth Cope, who developed the MERLIN analysis shown in Figure 23, Lizzie Wann whose project on zinc imidazolate contributed to the results shown in Figure 20; Björn Winkler, Keith Refson and Leila Rimmer who helped with the $\mathrm{Cu}_{2} \mathrm{O}$ calculations shown in Figure 21; and Prashant Selvaratnam who collaborated on the $\mathrm{YPO}_{4}$ study, Figure 18. Via our membership of the UK's HPC Materials Chemistry Consortium, which is funded by EPSRC (EP/F067496), some of this work made use of the facilities of HECToR, the UK's national high-performance computing service, which is provided by UoE HPCx Ltd at the University of 
Edinburgh, Cray Inc and NAG Ltd, and funded by the Office of Science and Technology through EPSRC's High End Computing Programme. Other calculations were performed on the CamGrid campus grid of the University of Cambridge.

\section{A. SOME RESULTS INVOLVING NORMAL MODE COORDINATES}

\section{A.1 Simple lattice sums}

In this appendix, we make use of the following lattice sums:

$$
\frac{1}{N} \sum_{\mathbf{R}} \exp (i \mathbf{k} \cdot \mathbf{R})=\delta_{\mathbf{k}, 0}
$$

where $\mathbf{k}$ is a wave vector, and $\mathbf{R}$ is a lattice vector. Similarly

$$
\frac{1}{N} \sum_{\mathbf{G}} \exp (i \mathbf{G} \cdot \mathbf{r})=\delta_{\mathbf{r}, 0}
$$

where $\mathbf{r}$ is a vector in real space, and $\mathbf{G}$ is a reciprocal lattice vector. Both results follow from the fact that the arguments of the summation care complex numbers with real and imaginary components varying between \pm 1 and $\pm i$ respectively, and with an inifinite number of terms the summations will be zero unless the arguments of the exponential are zero in value.

\section{A.2 Harmonic kinetic energy}

The kinetic energy of a single atom can be written as

$$
\frac{1}{2} m_{j}\left|\dot{\mathbf{u}}_{j \ell}\right|^{2}=\frac{m_{j}}{2 N} \sum_{\substack{\mathbf{k}, \mathbf{k}^{\prime} \\ \lambda, \lambda^{\prime}}} \omega_{\mathbf{k}, \lambda} \omega_{\mathbf{k}^{\prime}, \lambda^{\prime}} \mathbf{e}(j, \mathbf{k}, \lambda) \cdot \mathbf{e}^{*}\left(j, \mathbf{k}^{\prime}, \lambda^{\prime}\right) \exp \left(i\left(\mathbf{k}-\mathbf{k}^{\prime}\right) \cdot \mathbf{r}_{j \ell}\right) Q_{\mathbf{k}, \lambda} Q_{\mathbf{k}^{\prime}, \lambda^{\prime}}^{*}
$$

The total kinetic energy involves the sum over all atoms, that is, over all all values of the pair $j, \ell$. The sum over $\ell$ is the sum over all lattice points. The sum rule of equation (A.1) means that the sum is equal to zero unless $\mathbf{k}=\mathbf{k}^{\prime}$. In this case, the orthonormality of the mode eigenvectors also requires that the terms are zero unless $\lambda=\lambda^{\prime}$. Thus the kinetic energy of the crystal quickly reduces to the form given in equation (3.6).

\section{A.3 Harmonic potential energy}

The potential energy of a single atom can be written as

$$
\begin{aligned}
\frac{1}{2} \sum_{\substack{j, j^{\prime} \\
\ell, \ell^{\prime}}} \mathbf{u}_{j \ell}^{T} \cdot \Phi_{\ell, \ell^{\prime}}^{j, j^{\prime}} \cdot \mathbf{u}_{j^{\prime} \ell^{\prime}} & =\frac{1}{2 N} \sum_{\substack{j, j^{\prime} \\
\ell, \ell^{\prime}}} \frac{1}{\sqrt{m_{j} m_{j^{\prime}}}} \sum_{\substack{\mathbf{k}, \mathbf{k}^{\prime} \\
\lambda, \lambda^{\prime}}} \mathbf{e}^{\mathrm{T}}(j, \mathbf{k}, \lambda) \cdot \Phi_{\ell, \ell^{\prime}}^{j, j^{\prime}} \cdot \mathbf{e}\left(j^{\prime}, \mathbf{k}^{\prime}, \lambda^{\prime}\right) \\
& \times \exp \left(i\left(\mathbf{k} \cdot \mathbf{r}_{j \ell}+\mathbf{k}^{\prime} \cdot \mathbf{r}_{j^{\prime} \ell^{\prime}}\right)\right) \times Q_{\mathbf{k}, \lambda} Q_{\mathbf{k}^{\prime}, \ell^{\prime}}
\end{aligned}
$$

The sum rules again give the condition that $\mathbf{k}=-\mathbf{k}^{\prime}$. In this case, a large part of this equation has the form of the dynamical matrix:

$$
\begin{aligned}
\frac{1}{2} \sum_{\substack{j, j^{\prime} \\
\ell, \ell^{\prime}}} \mathbf{u}_{j \ell}^{T} \cdot \Phi_{\ell, \ell^{\prime}}^{j, j^{\prime}} \cdot \mathbf{u}_{j^{\prime} \ell^{\prime}} & =\frac{1}{2 N} \sum_{\substack{j, j^{\prime} \\
\ell, \ell^{\prime}}} \sum_{\mathbf{k}, \lambda} \mathbf{e}^{T}(j, \mathbf{k}, \lambda) \cdot \mathbf{D}(\mathbf{k}) \cdot \mathbf{e}\left(j^{\prime},-\mathbf{k}, \lambda\right) Q_{\mathbf{k}, \lambda} Q_{-\mathbf{k}, \lambda} \\
& =\frac{1}{2} \sum_{\mathbf{k}, \lambda} \omega_{\mathbf{k}, \lambda}^{2} Q_{\mathbf{k}, \lambda} Q_{-\mathbf{k}, \lambda}
\end{aligned}
$$




\section{B. PHONON PARTITION FUNCTION AND THERMODYNAMIC QUANTITIES}

\section{B.1 Partition function}

Consider a system that can exist in any number of states, with each state labelled $j$ having energy $E_{j}$. The mean energy can be written as

$$
\langle E\rangle=\frac{\sum_{j} E_{j} \exp \left(-\beta E_{j}\right)}{\sum_{j} \exp \left(-\beta E_{j}\right)}
$$

where $\beta=k_{\mathrm{B}} T$, and is called the inverse temperature. The denominator has a special function in statistical mechanics, and is called the partition function:

$$
\mathcal{Z}=\sum_{j} \exp \left(-\beta E_{j}\right)
$$

We can write relationships between mean quantities and differentials of $\mathcal{Z}$; for example, the mean energy can be written as

$$
\langle E\rangle=-\frac{1}{\mathcal{Z}} \frac{\partial \mathcal{Z}}{\partial \beta}
$$

\section{B.2 Partition function for phonons and the Bose-Einstein distribution}

In the case of quantised lattice vibrations, the energy of any state is simply the number of phonons multiplied by the energy of a single quantum: $E_{n}=\hbar \omega .{ }^{22}$ Thus the partition function is

$$
\mathcal{Z}=\sum_{n} \exp (-n \beta \hbar \omega)
$$

Since we can write $x=\exp (-\beta \hbar \omega)$, and thus $\exp (-n \beta \hbar \omega)=x^{n}$, we make use of the result

$$
\sum_{n} x^{n}=\frac{1}{1-x}
$$

to write the partition function for phonons as

$$
\mathcal{Z}=\frac{1}{1-\exp (-\beta \hbar \omega)}
$$

Using equation (B.3) we have

$$
\langle E\rangle=-(1-\exp (-\beta \hbar \omega)) \times \frac{-\hbar \omega \exp (-\beta \hbar \omega)}{(1-\exp (-\beta \hbar \omega))^{2}}=\frac{\hbar \omega \exp (-\beta \hbar \omega)}{1-\exp (-\beta \hbar \omega)}=\frac{\hbar \omega}{\exp (\beta \hbar \omega)-1}
$$

From the fact that $\langle E\rangle=\langle n\rangle \hbar \omega$, we have

$$
\langle n\rangle=\frac{1}{\exp (\beta \hbar \omega)-1}
$$

This is the Bose-Einstein distribution.

\footnotetext{
22 Here we ignore the zero point energy; there is no effect in so doing.
} 


\section{References}

[1] M.T. Dove. Introduction to Lattice Dynamics. Cambridge University Press (1993)

[2] M.T. Dove. Structure and Dynamics: An Atomic View of Materials. Oxford University Press (2003)

[3] A. Einstein. The motion of elements suspended in static liquids as claimed in the molecular kinetic theory of heat. Annalen der Physik 17, 549-560 (1905).

[4] A. Einstein. A new determination of the molecular dimensions. Annalen der Physik 19, 289-306 (1906).

[5] A. Einstein. The Planck theory of radiation and the theory of specific heat. Annalen der Physik 22, 180-190 (1907).

[6] M. Born and Th. von Kàrmàn. On fluctuations in spatial grids. Physikalische Zeitschrift 13, 297 309 (1912).

[7] M. Born and Th. von Kàrmàn. On the distribution of natural vibrations of spot-lattices. Physikalische Zeitschrift 13, 297-309 (1912).

[8] P. Debye. Interference of X-rays and thermal motion. Annalen der Physik 43, 49-95 (1914).

[9] I. Waller. On the question of the effect of thermal motion on X-ray interference. Zeitschrift fur Physik 17, 398-408 (1923).

[10] W. Cochran Crystal stability and the theory of ferroelectricity. Advances in Physics 9, 387-423 (1960).

[11] P. Debye. The theory of specific heat. Annalen der Physik 39, 789-839 (1912).

[12] Y. Endoh, G. Shirane and J. Skalyo Jr. Lattice dynamics of solid neon at 6.5 and 23.7 K. Physical Review B 11, 1681-1688 (1975).

[13] R.A. Cowley, A.D.B. Woods and G. Dolling. Crystal dynamics of potassium. I. Pseudopotential analysis of phonon dispersion curves at 9 K. Physical Review 150, 487-494 (1966).

[14] G. Raunio, L. Almqvist and R. Stedman. Phonon dispersion relations in NaCl. Physical Review 178, 1496-1501 (1969).

[15] E.R. Cowley and A.K. Pant. Lattice dynamics of calcite. Physical Review B 8, 4795-4800 (1973).

[16] S.L. Chaplot, G.J. McIntyre, A. Mierzejewsk and G.S. Pawley. Structure of 1,3,5-trichloro-2,4,6trifluorobenzene. Acta Crystallographica B 37, 1896-1900 (1981)

[17] M.T. Dove, B.M. Powell, G.S. Pawley, S.L. Chaplot and A. Mierzejewski. Inelastic neutron scattering determination of phonon dispersion curves in the molecular crystal sym- $\mathrm{C}_{6} \mathrm{~F}_{3} \mathrm{Cl}_{3}$. Journal of Chemical Physics 90, 1918-1923 (1989).

[18] J.D. Gale and A.L. Rohl. The General Utility Lattice Program (GULP). Molecular Simulation 29, 291-341 (2003).

[19] P. Mogilevsky, E.B. Zaretsky, T.A. Parthasarathy and F. Meisenkothen. Composition, lattice parameters, and room temperature elastic constants of natural single crystal xenotime from Novo Horizonte. Physics and Chemistry of Minerals 33, 691-698 (2006).

[20] K. Trachenko, M.T. Dove and E.K.H. Salje. Reply to comment on 'Large swelling and percolation in irradiated zircon'. Journal of Physics: Condensed Matter 15, 6457-6471 (2003).

[21] K.S. Park, Z. Ni, A.P. Cote, J.Y. Choi, R. Huang, F.J. Uribe-Romo, H.K. Chae, M. OÕKeeffe and O.M. Yaghi. Exceptional chemical and thermal stability of zeolitic imidazolate frameworks. Proceedings of the National Academy of Sciences 103, 10187-10191 (2006).

[22] N.L. Allinger, Y.H. Yuh, and J.H. Lii. Molecular mechanics. The MM3 force field for hydrocarbons. 1. Journal of the American Chemical Society 111, 8551-8566 (1989).

[23] D.E. Williams. Improved intermolecular force field for molecules containing $\mathrm{H}, \mathrm{C}, \mathrm{N}$, and $\mathrm{O}$ atoms, with application to nucleoside and peptide crystals. Journal of Computational Chemistry 22, 1154-1166 (2001).

[24] DALTON, a molecular electronic structure program, Release 2.0 (2005), see http://daltonprogram.org/ 
[25] A.J. Stone and M. Alderton. Distributed multipole analysis. Molecular Physics 56, 1047-1064 (1985).

[26] R.M. Martin. Electonic Structure, Basic Theory and Practical Methods. Cambridge University Press (2004).

[27] G.J. Ackland, M.C. Warren and S.J. Clark. Practical methods in ab initio lattice dynamics. Journal of Physics: Condensed Matter 9, 7861-7872 (1997).

[28] G.D. Barrera, J.A.O. Bruno, T.H.K. Barron and N.L. Allan

[29] V. Heine, P.R.L. Welche and M.T. Dove. Geometrical origin and theory of negative thermal expansion in framework structures. Journal of the American Ceramic Society 82, 1793-802 (1999).

[30] K-P. Bohnem, R. Hei, L. Pintschovius, A. Soon and C. Stampf. Ab initio lattice dynamics and thermal expansion of Cu2O. Physical Review B 80, 134304 (2009).

[31] R.I. Bewley, R.S. Eccleston, K.A. McEwen, S.M. Hayden, M.T. Dove, S.M. Bennington, J.R. Treadgold and R.L.S. Coleman. MERLIN, a new high count rate spectrometer at ISIS. Physica B 385-386, 1029-1031 (2006).

[32] I. Fischer, A. Bosak, and M. Krisch. Single-crystal lattice dynamics derived from polycrystalline inelastic X-ray scattering spectra. Physical Review B 79, 134302 (2009).

[33] T.D. Archer, S.E.A. Birse, M.T. Dove, S.A.T. Redfern, J.D. Gale and R.T. Cygan. An interatomic potential model for carbonates allowing for polarization effects. Physics and Chemistry of Minerals 30, 416-424 (2003). 